Draft Version OCtOBER 30, 2018

Preprint typeset using $\mathrm{LAT}_{\mathrm{E} X} \mathrm{X}$ style emulateapj v. 03/07/07

\title{
STOCHASTIC ACCELERATION AND THE EVOLUTION OF SPECTRAL DISTRIBUTIONS IN SSC SOURCES: A SELF CONSISTENT MODELING OF BLAZARS' FLARES
}

\author{
A. Tramacere \\ ISDC, University of Geneva, Chemin d'Ecogia 16, Versoix, CH-1290, Switzerland \\ E. MASSARO \\ Dipartimento di Fisica, Università La Sapienza, Piazzale A. Moro 2, I-00185 Roma, Italy \\ A. M. TAYLOR \\ ISDC, University of Geneva, Chemin d'Ecogia 16, Versoix, CH-1290, Switzerland \\ Draft version October 30, 2018
}

\begin{abstract}
The broad-band spectral distributions of non-thermal sources, such as those of several known blazars, are well described by a log-parabolic fit. The second degree term in these fits measures the curvature in the spectrum. In this paper we investigate whether the curvature parameter observed in the spectra of the synchrotron emission can be used as a fingerprint of stochastic acceleration.

As a first approach we use the multiplicative Central Limit theorem to show how fluctuations in the energy gain result in the broadening of the spectral shape, introducing a curvature into the energy distribution. Then, by means of a Monte-Carlo description, we investigate how the curvature produced in the electron distribution is linked to the diffusion in momentum space. To get a more generic description of the problem we turn to the diffusion equation in momentum space. We first study some "standard" scenarios, in order to understand the conditions that make the curvature in the spectra significant, and the relevance of cooling during the acceleration process. We try to quantify the correlation between the curvature and the diffusive process in the pre-equilibrium stage, and investigate how the transition between the Klein-Nishina and the Thompson regime, in Inverse Compton cooling, determine the curvature in the distribution at equilibrium. We apply these results to some observed trends, such as the anticorrelation between the peak energy and the curvature term observed in the spectra of Mrk 421, and a sample of BL Lac objects whose synchrotron emission peaks at X-ray energies

Subject headings: acceleration mechanisms: statistical - galaxies: active - galaxies: BL Lacertae objects - galaxies: BL Lacertae objects (Mrk 421, Mrk 501, 1H 1426+428, 1ES 1959+650, Mrk 180, PKS 0548-322)
\end{abstract}

\section{INTRODUCTION}

A defining feature of the non-thermal emission from different types of galactic and extragalactic sources is that their spectra are described by a power-law (PL) over a broad photon energy range. In several sources, however, their spectra show significant curvature that is typically milder than that expected from an exponential cut-off. In previous papers Massaro et al. (2004, 2006) discussed the curvature observed in the broad band X-ray spectra of the two well known HBL (Highenergy peaked BL Lac) objects Mkn 421 and Mkn 501. The basic idea was that this curvature was not simply the result of radiative cooling of high energy electrons, responsible of the synchrotron and inverse Compton emission, but that it was essentially related to the acceleration mechanism. Massaro et al. (2004) showed that curved spectral distributions, in particular logparabolic (i.e. log-normal) ones, develop when the acceleration probability is a decreasing function of the electron energy. In subsequent works, through the analysis of a large collection of X-ray observations of Mkn 421, Tramacere et al. (2007); Tramacere (2007);

Electronic address: andrea.tramacere@unige.ch
Tramacere et al. (2009) pointed out that the observed anticorrelation between the peak energy and the curvature measured in the synchrotron Spectral Energy Distribution (SED), could be used as a clear signature of a stochastic component in the acceleration process. Very recently, the log-parabolic law has been also applied to describe the spectral distribution and evolution of some Gamma-ray bursts (Massaro \& Grindlav 2011).

The principal aim of the present paper is to investigate this scenario by extracting information on the acceleration processes using the curvature parameter measured in the observed synchrotron and Inverse Compton (IC) spectra of Synchro-Self Compton (SSC) sources. We study the conditions in which the energy distributions of electrons, resulting from stochastic acceleration, can be approximated by a log-parabolic law and how its curvature evolves during their acceleration, and the role of IC cooling. We compare predictions from our theoretical descriptions with the curved spectra of some HBL objects.

In Sec. 2 we give an intuitive picture to take into account the effect of random fluctuations in the energy gain of particles and the role these play in determining the spectral curvature, as a consequence of the multiplicative 
central limit theorem, and compare these results with the analytical solution of the diffusion equation, in the "hardspheres" approximation. In Sec. 3 and Sec. 4 we give a more physical description of the problem, using first a Monte Carlo approach, and secondly by solving numerically the momentum diffusion equation. We discuss the evolution of the curvature in the electron distribution as a result of momentum-diffusion before equilibrium is reached, and the role that synchrotron and IC cooling processes play on reaching the equilibrium. In Sec. 5. we study the peak energy, fluxes, and curvature, trends in the SED of both the synchrotron and IC emission, looking for the fingerprints of the stochastic component. In Sec. 6, we show how our results can reproduce the spectral trends observed in a some HBLs, in particular we investigate the relation between the peak energy and the curvature, and between the peak energy and the peak flux. The good agreement between predictions and observed trends, confirms that the stochastic acceleration mechanism can play an important role in the physics of the blazars' jets and other SSC sources.

\section{THE LOG PARABOLA ORIGIN: ANALYTICAL APPROACH}

\subsection{Statistical description}

In the statistical picture, the change in energy of the particles at each acceleration step $n_{s}$ is expressed as

$$
\gamma_{n_{s}}=\varepsilon_{n_{s}} \gamma_{n_{s}-1}=\gamma_{n_{s}-1}\left(1+\Delta \gamma_{n_{s}-1} / \gamma_{n_{s}-1}\right)
$$

where $\gamma$ is the Lorentz factor of the particle and $\varepsilon$ is the fractional energy gain. We here investigate the role of fluctuations of $\varepsilon$, on the spectral shape of the accelerated particles. With this aim in mind, we express the energy gain fluctuations as

$$
\varepsilon=\bar{\varepsilon}+\chi
$$

where the random variable $\chi$ has a probability density function with zero mean value $(\langle\chi\rangle=0)$ and variance $\sigma_{\chi}^{2}$, and $\bar{\varepsilon}$ represents the systematic energy gain, that we treat as a non-random variable and the probability density function of $\varepsilon$ is defined on the range $\varepsilon \geq 0$. The particle energy at step $n_{s}$ can be expressed as:

$$
\gamma_{n_{s}}=\gamma_{0} \Pi_{i=1}^{n_{s}} \varepsilon_{i}
$$

where $\gamma_{0}$ is the initial energy of the particle. This equation clearly shows that the final energy distribution $(n(\gamma)=d N(\gamma) / d \gamma)$ will result from the product of the random variables $\varepsilon_{i}$. The determination of an analytic expression for the distribution resulting from the multiplication of generic random variable is not an easy task (Glen et al. 2004). Using the simplifying assumption that the particles are always accelerated, namely the acceleration probability, $P_{a}$, is set to unity and applying the multiplicative case of the central limit theorem (e.g. Cowan 1998) it is possible to show that the particle energies will be distributed as a log-normal law:

$$
n(\gamma)=\frac{N_{0}}{\gamma \sigma_{\gamma} \sqrt{(2 \pi)}} \exp \left[-(\ln \gamma-\mu)^{2} / 2 \sigma_{\gamma}^{2}\right]
$$

where $N_{0}$, is the total number of particles, $\mu=\langle\ln \gamma\rangle$, $\sigma_{\gamma}^{2}=\sigma^{2}(\ln \gamma)$. We can determine these two quantities by taking the logarithm of Eq. 3

$$
\begin{aligned}
\ln \gamma_{n_{s}} & =\ln \gamma_{0}+\sum_{i=1}^{n_{s}} \ln \left(\bar{\varepsilon}+\chi_{i}\right) \\
& =\ln \left(\gamma_{0} \bar{\varepsilon}^{n_{s}}\right)+\sum_{i=1}^{n_{s}} \ln \left(1+\frac{\chi_{i}}{\bar{\varepsilon}}\right) \\
& \approx \ln \left(\gamma_{0} \bar{\varepsilon}^{n_{s}}\right)+\sum_{i=1}^{n_{s}}\left(\frac{\chi_{i}}{\bar{\varepsilon}}-\frac{\chi_{i}^{2}}{2 \bar{\varepsilon}^{2}}\right)
\end{aligned}
$$

assuming that $\chi_{i} / \bar{\varepsilon}$ is not large. We obtain for the two parameters $\mu$ and $\sigma_{\gamma}$ :

$$
\begin{aligned}
\mu & =\ln \left(\gamma_{0}\right)+\mathrm{n}_{\mathrm{s}} \ln \bar{\varepsilon}+\mathrm{n}_{\mathrm{s}}\left[\left\langle\frac{\chi}{\bar{\varepsilon}}\right\rangle-\frac{1}{2}\left(\frac{\sigma_{\chi}}{\bar{\varepsilon}}\right)^{2}-\left\langle\frac{\chi}{2 \bar{\varepsilon}}\right\rangle^{2}\right] \\
\sigma_{\gamma}^{2} & =\mathrm{n}_{\mathrm{s}}\left[\left(\frac{\sigma_{\chi}}{\bar{\varepsilon}}\right)^{2}+\left(\frac{\sigma_{\chi}}{2 \bar{\varepsilon}}\right)^{4}+2\left(\frac{\sigma_{\chi}}{2 \bar{\varepsilon}}\left\langle\frac{\chi}{2 \bar{\varepsilon}}\right\rangle\right)^{2}\right]
\end{aligned}
$$

where we have ignored the covariance terms since we are assuming the energy gain at each acceleration step being independent on the one at the previous step. Remembering that $\langle\chi\rangle=0, \sigma_{\chi}=\sigma_{\varepsilon}$, and ignoring the 4-th order term, we can write:

$$
\begin{aligned}
\mu & =\ln \left(\gamma_{0}\right)+n_{s}\left[\ln \bar{\varepsilon}-\frac{1}{2}\left(\frac{\sigma_{\varepsilon}}{\bar{\varepsilon}}\right)^{2}\right] \\
\sigma_{\gamma}^{2} & \approx n_{s}\left(\frac{\sigma_{\varepsilon}}{\bar{\varepsilon}}\right)^{2}
\end{aligned}
$$

This equation shows that the variance increases linearly with the number of acceleration steps and it is proportional to $\sigma_{\varepsilon}{ }^{2}$. Substituting $\mu$ and $\sigma_{\gamma}$ into Eq. 4.

$$
n(\gamma)=\frac{N_{0}}{\gamma \sigma_{\gamma} \sqrt{(2 \pi)}} \exp \left[\frac{-\left(\ln \frac{\gamma}{\gamma_{0}}-n_{s}\left[\ln \bar{\varepsilon}-\frac{1}{2}\left(\frac{\sigma_{\varepsilon}}{\bar{\varepsilon}}\right)^{2}\right]\right)^{2}}{2 n_{s}\left(\frac{\sigma_{\varepsilon}}{\bar{\varepsilon}}\right)^{2}}\right]
$$

Hereafter we will consider decimal logarithms ( $\log \equiv$ $\log _{10}, c_{e}=1 / \log _{10} e \approx 2.3$ ), to make easier a comparison of the curvature results form this paper with those presented in observational papers. Taking the logarithm of Eq. 8, and substituting the parameters from Eq. 8 we obtain:

$\log n(\gamma)=K-\log \gamma-\frac{\left(c_{e} \log \frac{\gamma}{\gamma_{0}}-n_{s}\left[c_{e} \log \bar{\varepsilon}-\frac{1}{2}\left(\frac{\sigma_{\varepsilon}}{\bar{\varepsilon}}\right)^{2}\right]\right)^{2}}{c_{e} 2 n_{s}\left(\frac{\sigma_{\varepsilon}}{\bar{\varepsilon}}\right)^{2}}$

where $K$ includes all the constant factors. This is a logparabolic law with the curvature (2nd degree in $\log \gamma$ ) coefficient given by:

$$
r=\frac{c_{e}}{2 n_{s}\left(\frac{\sigma_{\varepsilon}}{\bar{\varepsilon}}\right)^{2}}
$$

The interesting physical insight of this equation is that the curvature of the particle energy distribution is inversely proportional to the acceleration steps $\left(n_{s}\right)$, and to the variance of the energy gain $\left(\sigma_{\varepsilon}^{2}\right)$. In the case of $P_{a}<1$, the distribution at step $n_{s}$ will be given by the convolution of different log-normal distributions for each acceleration step, with the distribution at $n_{s}$ broader than that at $n_{s}-1$ and containing fewer particles, as already noted in Peacock (1981).

Similar results are obtained considering a constant energy gain but a fluctuating number of acceleration steps. Assuming that after a time $t$ the probability distribution 
for the number of steps undergone by a particle is given by a Poisson law, it is possible to show that the energy distribution follows a log-parabola whose curvature term depends on the inverse of the mean number of steps multiplied by the duration of the acceleration process.

\subsection{Diffusion equation approach}

The above statistical description provides an intuitive link between the curvature in the energy distribution of accelerated particles and the presence of a randomization process, such as the dispersion in the energy gain or in the number of acceleration steps. However, this approach does not give a complete physical description of the processes responsible for the systematic and stochastic energy gain, ignoring other physical processes, such as the radiative cooling and injection rates, or the acceleration energy dependence, necessary to give a complete description of the particles energy distribution evolution. A physical self-consistent description of stochastic acceleration in a time-dependent fashion, can be achieved through a kinetic equation approach. Employing the quasi-linear approximation with the inclusion of momentum diffusion term (Ramaty 1979; Becker et al. 2006), the equation governing the temporal evolution of $n(\gamma)$ is:

$$
\begin{aligned}
\frac{\partial n(\gamma, t)}{\partial t} & =\frac{\partial}{\partial \gamma}\left\{-\left[S(\gamma, t)+D_{A}(\gamma, t)\right] n(\gamma, t)\right\} \\
& +\frac{\partial}{\partial \gamma}\left\{D_{p}(\gamma, t) \frac{\partial n(\gamma, t)}{\partial \gamma}\right\}-\frac{n(\gamma, t)}{T_{e s c}(\gamma)}+Q(\gamma, t)
\end{aligned}
$$

where $D_{p}(\gamma, t)$ is the momentum diffusion coefficient, $D_{A}(\gamma, t)=(2 / \gamma) D_{p}(\gamma, t)$ is the average energy change term resulting from the momentum-diffusion process, and $S(\gamma, t)=-C(\gamma, t)+A(\gamma, t)$ is an extra term describing systematic energy loss $(C)$ and/or gain $(A)$, and $Q(\gamma, t)$ is the injection term. In the standard diffusive shock acceleration scenario, there are several possibilities for which one can expect that energy gain fluctuations will occur, due to the momentum diffusion term. In particular, for the case of a turbulent magnetized medium, the advection of particles towards the shock due to pitch angle scattering may be accompanied by stochastic momentum diffusion mechanism. In this scenario, particles embedded in a magnetic field with both an ordered $\left(B_{0}\right)$ and turbulent $(\delta B)$ component, exchange energy with resonant plasma waves, and the related diffusion coefficient is determined by the spectrum of the plasma waves. Following the approach of Becker et al. (2006) we describe the energy distribution $W(k)$ in terms of the wave number $k=2 \pi / \lambda$ with a power-law :

$$
W(k)=\frac{\delta B(k)^{2}}{8 \pi}=\frac{\delta B\left(k_{0}\right)^{2}}{8 \pi}\left(\frac{k}{k_{0}}\right)^{-q} .
$$

with $q=2$ for the "hard-sphere" spectrum, $q=5 / 3$ for the Kolmogorov spectrum, and $q=3 / 2$ for the Kraichnan spectrum, the total energy density in the fluctuations being

$$
U_{\delta B}=\int_{k_{0}}^{k_{\max }} W(k) d k
$$

Under these assumptions the momentum-diffusion coefficient reads (O'Sullivan et al. 2009):

$$
D_{p} \approx \beta_{A}^{2}\left(\frac{\delta B}{B_{0}}\right)^{2}\left(\frac{\rho_{g}}{\lambda_{\max }}\right)^{q-1} \frac{p^{2} c^{2}}{\rho_{g} c}
$$

where $\beta_{A}=V_{A} / c$ and $V_{A}$ is the Alfven waves velocity, $\rho_{g}=p c / q B$ is the Larmor radius, and $\lambda_{\max }$ is the maximum wavelength of the Alfven waves spectrum. The acceleration time for particles with Lorentz factor $\gamma$, whose Larmor radii resonate with one particular magnetic field turbulence length-scale, is dictated by the momentum diffusion coefficient $\left(D_{p}\right)$ as,

$$
t_{\mathrm{acc}} \approx \frac{p^{2}}{D_{p}}=\left.\frac{\rho_{g}\left(\gamma_{0}\right)}{c \beta_{A}^{2}}\left(\frac{B_{0}^{2}}{\delta B^{2}}\right)\right|_{\gamma_{0}}\left(\frac{\gamma}{\gamma_{0}}\right)^{2-q}
$$

The spatial diffusion coefficient relates to the momentum diffusion coefficient through the relation, $D_{x} D_{p} \approx p^{2} \beta_{\mathrm{A}}^{2}$ (Skilling 1975), hence the escape time of the particles from the acceleration region of size $R$, depends on the spatial diffusion coefficient through the relation,

$$
t_{\mathrm{esc}} \approx \frac{R^{2}}{D_{x}} \approx \frac{R^{2}}{\left(c \beta_{\mathrm{A}}\right)^{2} t_{\mathrm{acc}}}
$$

The coefficients in Eq. 12, and their related time scales, can be expressed as a power-law in terms of the Lorentz factor $(\gamma)$ :

$$
\left\{\begin{array}{lll}
D_{p}(\gamma)=D_{p 0}\left(\frac{\gamma}{\gamma_{0}}\right)^{q}, & t_{D}=\frac{1}{D_{p 0}}\left(\frac{\gamma}{\gamma_{0}}\right)^{2-q} \\
D_{A}(\gamma)=2 D_{p 0}\left(\frac{\gamma}{\gamma_{0}}\right)^{q-1}, & t_{D A}=\frac{1}{2 D_{p 0}}\left(\frac{\gamma}{\gamma_{0}}\right)^{2-q} \\
A(\gamma) & =A_{p 0} \gamma, & t_{A}=\frac{1}{A_{0}}
\end{array}\right.
$$

where $D_{p 0}$, and $A_{0}$ have the dimension of the inverse of a time. Analytical solutions of the diffusion equation for relativistic electrons are frequently discussed in the literature since the early work by Kardashev (1962), in particular for the case of the "hard-sphere" approximation. Neglecting the $S$ and $T_{\text {esc }}$ terms in Eq. 12 , and using a mono-energetic and instantaneous injection $\left(n(\gamma, 0)=N_{0} \delta\left(\gamma-\gamma_{0}\right)\right)$, the solution of the diffusion equation is (Melrose 1969; Kardashev 1962):

$n(\gamma, t)=\frac{N_{0}}{\gamma \sqrt{4 \pi D_{p 0} t}} \exp \left\{-\frac{\left[\ln \left(\gamma / \gamma_{0}\right)-\left(A_{p 0}-D_{p 0}\right) t\right]^{2}}{4 D_{p 0} t}\right\}$,

ie. a log-parabolic distribution, whose curvature term is:

$$
r=\frac{c_{e}}{4 D_{p 0} t} \propto \frac{1}{D_{p 0} t}
$$

This result is fully consistent with that found in the statistical description, indeed Eq. 18 and Eq. 8 have the same functional form in both the statistical and in the diffusion equation scenario, with $t$ playing the role of $n_{\mathrm{s}}$, $D_{p 0}$ the role of the variance of the energy gain $\left(\sigma_{\varepsilon}^{2}\right)$, and $A_{p 0}$ the role of $\log \bar{\varepsilon}$. Hence we can write:

$$
D_{p 0} \propto\left(\frac{\sigma_{\varepsilon}}{\bar{\varepsilon}}\right)^{2}
$$

It is interesting to note, that in the case of the "hardsphere" approximation, the curvature term is simply dictated by the ratio of the diffusive acceleration time $\left(t_{D}\right)$ to the evolution time $(t)$. 


\section{NUMERICAL APPROACH: MONTE-CARLO} SIMULATION WITH MAGNETIC TURBULENCE

In this section we demonstrate explicitly how the introduction of energy fluctuations leads to curved spectral distributions of particles. This is carried out using a Monte-Carlo (MC) approach.

In our simulations, we considered $10^{5}$ particles injected into the system with a cold mono-energetic distribution of Lorentz factors, with $\gamma_{0}=1$. To compare these results with the ones presented in Sec. 2, we remind the reader that, in the MC approach, the duration of the acceleration process $t$ is the equivalent of the number of acceleration step $\left(n_{s}\right)$ used in the statistical picture, and that the probability of the particle to be up-scattered or downscattered in the $\mathrm{MC}$ realizations, can be expressed in the statistical approach as $P(\varepsilon>1)$ and $P(\varepsilon<1)$, respectively. The scattering probability of the particles is dictated by the intensity of resonant waves in the turbulent magnetic power spectrum. As a working hypothesis we assume that particles interact with a turbulent magnetic field whose power spectrum is expressed by Eq. 12 In each scattering, the particles have probability $\left(1+\beta_{\mathrm{A}}\right) / 2$ of being up-scattered, and probability $\left(1-\beta_{\mathrm{A}}\right) / 2$ of being down-scattered. The energy dispersion of the particle due to resonant scattering with Alfven waves will be $\left\langle\Delta E^{2}\right\rangle \propto\left(E \beta_{\mathrm{A}}\right)^{2} t$, where $E=m_{e} c^{2} \gamma$. Using the very good approximation for the variance of the product of $n$ uncorrelated random variables (Goodman 1962):

$$
\sigma^{2}\left(\Pi x_{i}\right)=\Pi\left\langle x_{i}\right\rangle^{2} \Sigma\left(\frac{\sigma_{x_{i}}^{2}}{\left\langle x_{i}\right\rangle^{2}}\right)
$$

and plugging Eq. 2 into the equation above, we get:

$$
\left\langle\Delta E^{2}\right\rangle \propto\left(E \beta_{\mathrm{A}}\right)^{2} t \propto \gamma_{0}^{2} \sigma^{2}\left(\Pi \varepsilon_{i}\right)=\left(\gamma_{0} \bar{\varepsilon}^{n_{s}}\right)^{2} n_{s} \frac{\sigma_{\varepsilon}^{2}}{\bar{\varepsilon}^{2}}
$$

since $E$ is the particle energy at time $t$ (namely step $n_{s}$ ), we have $E^{2}=\left(m_{e} c^{2} \gamma_{0} \bar{\varepsilon}^{n_{s}}\right)^{2}$, from which follows:

$$
\beta_{\mathrm{A}}^{2} \propto\left(\frac{\sigma_{\varepsilon}}{\bar{\varepsilon}}\right)^{2}
$$

In the following two sections (Sec. 3.1 and, Sec 3.2) we study the consequences of the structure in the magnetic turbulence, on the evolution of the particle spectra, following their stochastic acceleration in the turbulent field.

\subsection{Hard-Sphere Turbulence}

Under the "hard-sphere" approximation $(q=2)$, the spatial diffusion coefficient does not depend on the particle energy, since the exponent of Eq. 15 is $q-2=0$. Only three independent parameters exist in this description: the scattering time, the escape time and the velocity of the scatterers. The spectra are purely determined by how many scatterings have been able to occur, the velocity of the scatterer, and what fraction of the injected particles have escaped out of the acceleration region. The scattering time relates to the spatial diffusion coefficient by $t_{\text {scat }} \approx D_{x} / c$. Similarly, the resulting acceleration time relates to the spatial diffusion coefficient by $t_{\mathrm{acc}} \approx D_{x} / \beta_{\mathrm{A}}^{2} c \approx t_{\mathrm{A}} / \beta_{\mathrm{A}}^{2}$. Thus, for "hard sphere" turbulence, the scattering and acceleration time scales are independent of the particle energy (since there is equal energy density of scatterers which particles of all energies may resonantly scatter with).
The left-hand panel in Fig. 11 shows the resulting instantaneous evolution of spectra for the "hard sphere" turbulence. The log-parabolic shape is maintained along the entire acceleration process, as shown by the solid lines representing the fit of the $\mathrm{MC}$ distributions by means of the law in Eq. 9. The evolution of the curvature parameter, obtained from the $r$ in the log-parabolic fit, and plotted in Fig. 2 with the red dashed line, clearly shows the trend due to the momentum diffusion, in agreement with the prediction from Eq. 19 (blue line in the plot) demonstrating the connection between $D_{p 0}, \frac{\sigma_{\varepsilon}}{\bar{\varepsilon}}$, and $\beta_{\mathrm{A}}$.

\subsection{Soft turbulence spectra}

To account for the effects of turbulent magnetic field spectra softer than the "hard-sphere" case, we also consider acceleration in Kolmogorov and Kraichnan type turbulence. We have therefore to include a fourth parameter in the $\mathrm{MC}$ simulation, in addition to the three considered above: the turbulent field spectral slope $q$ (see Eq. 12).

The right-hand plot in Fig. 1 shows the evolution of spectra for the "Kolmogorov" turbulence case. Similar spectra were obtained by Lemoine \& Pelletier (2003), and O'Sullivan et al. (2009), who integrated the trajectories of charged particles in a turbulent magnetic field embedded in a fluid. The results are compared to the quasilinear theory results, Becker et al. (2006) (solid lines in Fig. 11). We can identify two phases in the temporal evolution. In the first phase, the spectral energy distributions are more symmetric, and the curvature evolves as in the $q=2$ case, while in the second phase they develop a low-energy power-law tail. Fig. 2] shows that, for the Kolmogorov (green line) and the Kraichnan (black line) turbulence, $r$ is systematically larger compared to the "hard-sphere" case (red line), and that for $t \gtrsim 2 \times t_{a c c}, r$ approaches to an asymptotic value $(r \approx 1.2$ and $r \approx 1.5$, for $q=5 / 3$ and $q=3 / 2$ respectively) ruled by the exponential cut-off in the equilibrium distribution.

\section{NUMERICAL APPROACH: DIFFUSION EQUATION WITH STOCHASTIC COMPONENT AND LOSSES}

Both MC approach and statistical description are able to explain the link between the curvature in the energy distribution of accelerated particles and the presence of a stochastic energy gain term. In order to incorporate a more complete description, taking into account the competition between radiative losses and acceleration, and its influence on the curvature, we use the diffusion equation approach, already outlined in Sec 2.2, by inserting into Eq. 12 a cooling term for the synchrotron and IC radiative losses. Following Moderski et al. (2005) we can write:

$$
\begin{aligned}
\left|\dot{\gamma}_{\text {synch }}\right| & =\frac{4 \sigma_{T} c}{3 m_{e} c^{2}} \gamma^{2} U_{B}=C_{0} \gamma^{2} U_{B} \\
\left|\dot{\gamma}_{I C}\right| & =\frac{4 \sigma_{T} c}{3 m_{e} c^{2}} \gamma^{2} \int f_{K N}\left(4 \gamma \epsilon_{0}\right) \epsilon_{0} n_{p h}\left(\epsilon_{0}\right) d \epsilon_{0}=C_{0} \gamma^{2} F_{K N}(\gamma) \\
C(\gamma) & =\left|\dot{\gamma}_{\text {synch }}\right|+\left|\dot{\gamma}_{I C}\right|=C_{0} \gamma^{2}\left(U_{B}+F_{K N}(\gamma)\right)
\end{aligned}
$$

where $U_{B}=B^{2} / 8 \pi$, is the energy density of the magnetic field, $\epsilon_{0}=h \nu_{0} / m_{e} c^{2}$ is the IC seed photon energy in units of $m_{e} c^{2}, n_{p h}\left(\epsilon_{0}\right)$ is the number density 

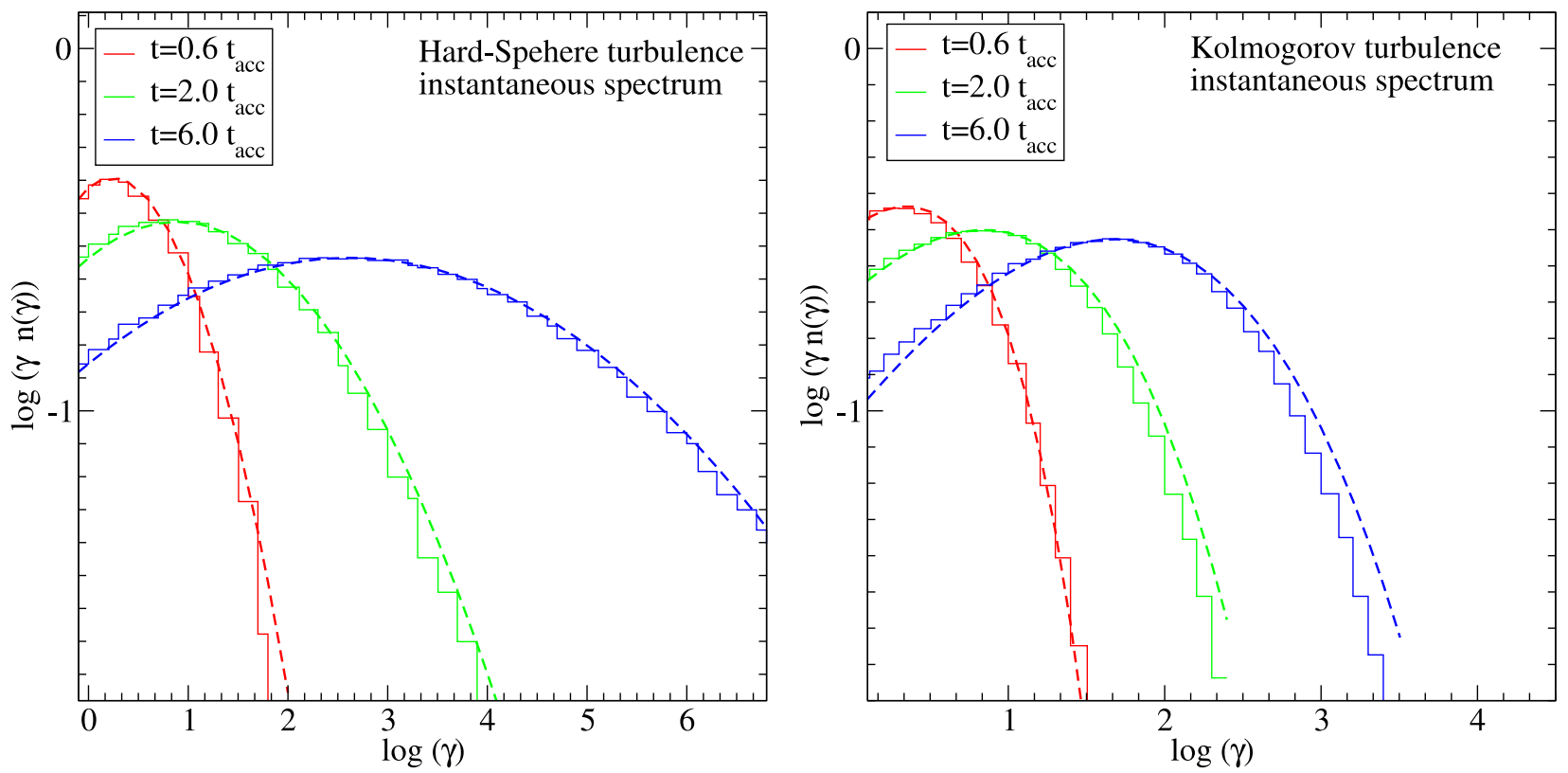

FIG. 1.- Plots showing the Monte Carlo results. For comparison, the results obtained using an analytic description given in Becker et al. (2006) (dashed line, right panel) and a log-parabolic function (dashed line, left panel), are shown.

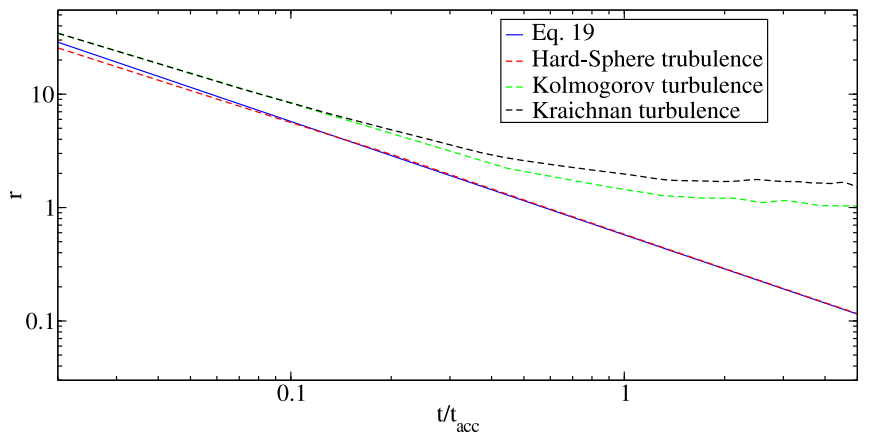

FIG. 2.- The curvature parameters of the energy distribution of accelerated electrons in the shown in Fig 1 In the case of $q=2$ (red line), the trend is consistent with the "hard-spheres" prediction (blue line). In the case of Kolmogorov (green line) and Kraichnan (black line) turbulence, the trend predicts larger values compared to the "hard-spheres" prediction, and $r$ approaches an asymptotic value dictated by the exponential cut-off in the equilibrium distribution.

of IC seed photons with the corresponding photon energy density $U_{p h}=m_{e} c^{2} \int \epsilon_{0} n_{p h}\left(\epsilon_{0}\right) d \epsilon_{0}$. The function $f_{K N}$ results from the analytical integration of the Jones (1968) Compton kernel, fully taking into account KleinNishina (KN) effects for an isotropic seed photon field (see Moderski et al. 2005, appendix C), and $F_{K N}(\gamma)$ represents its convolution with the seed photon field. We remark that $F_{K N}$ plays a crucial role in the cooling process, depending both on the IC regime (Thomson (TH) limit for $4 \gamma \epsilon_{0}<<1$, KN limit for $4 \gamma \epsilon_{0}>>1$ ), and on $\epsilon_{0} n_{p h}\left(\epsilon_{0}\right) \propto B^{2} / R^{2}$.

Since analytical solutions are possible only for a limited number of cases, to follow the complex dependence of the IC cooling term on $n_{p h}\left(\epsilon_{0}\right)$ in a self-consistent way, we must solve the diffusion equation numerically. For this purpose we further developed the numerical code (Tramacere et al. 2009; Tramacere 2007) used to compute numerically the synchrotron and IC emission, and introduced it into the numerical solution of the diffusion equation. In the numerical calculations, we adopted the method proposed by Chang \& Cooper(1970) and used the numerical recipe given by Park \& Petrosian (1996). This is a finite difference scheme based on the centered difference of the diffusive term, employing weighted differences for the advective term. We use a 5000 points energy grid over the range $1.0 \leqslant \gamma \leqslant 10^{9}$, and a time grid is finely tuned to have a temporal mesh several orders of magnitude smaller than typical cooling and acceleration time scales. The results from our code were compared, when possible, with known analytical solutions, and always found good agreement.

\subsection{Physical set-up: the relations between $D_{p}$, and $t_{D}$ with $\gamma_{\max }$ and $R$}

We study the evolution of $n(\gamma)$ and of the curvature term in an homogeneous spherical geometry, with radius $R$ and an entangled coherent magnetic field $B$ and a turbulent component $\delta B$, in the two cases of impulsive and continuous injection with a quasi mono-energetic source function $Q\left(\gamma_{i n j}, t\right)$ normalised to have a fixed energy input rate:

$$
L_{i n j}=\frac{4}{3} \pi R^{3} \int \gamma_{i n j} m_{e} c^{2} Q\left(\gamma_{i n j}, t\right) d \gamma_{i n j} \quad(e r g / s)
$$

In our approach we don't distinguish the acceleration region from the radiative one, and during the acceleration process we take into account both synchrotron and IC cooling. According to Eq. 14, to determine the order of magnitude of $D_{p}$ we assume $1>\delta B / B \simeq 0.1-0.01$ and require Alfven waves to be at least mildly relativistic, with $\beta_{A} \simeq 0.1-0.5$, and their maximum wavelength to be much smaller than the accelerator size $\left(\lambda_{\max }<\right.$ $R$ ). To study the effect of IC cooling on the evolution of $n(\gamma)$, we consider two different sizes of the acceleration region, a compact one $\left(R=5 \times 10^{13} \mathrm{~cm}\right)$, and a larger one $\left(R=1 \times 10^{15} \mathrm{~cm}\right)$. With this choice of accelerator size we set $\lambda_{\max } \approx 10^{12} \mathrm{~cm}$. We stress that the choice of $\lambda_{\max }$ constrains the accelerative upper limit through $\rho_{g}<\lambda_{\max }$ leading to $\gamma_{\max }<\left(\lambda_{\max } q B\right) / m_{e} c^{2}$, since 

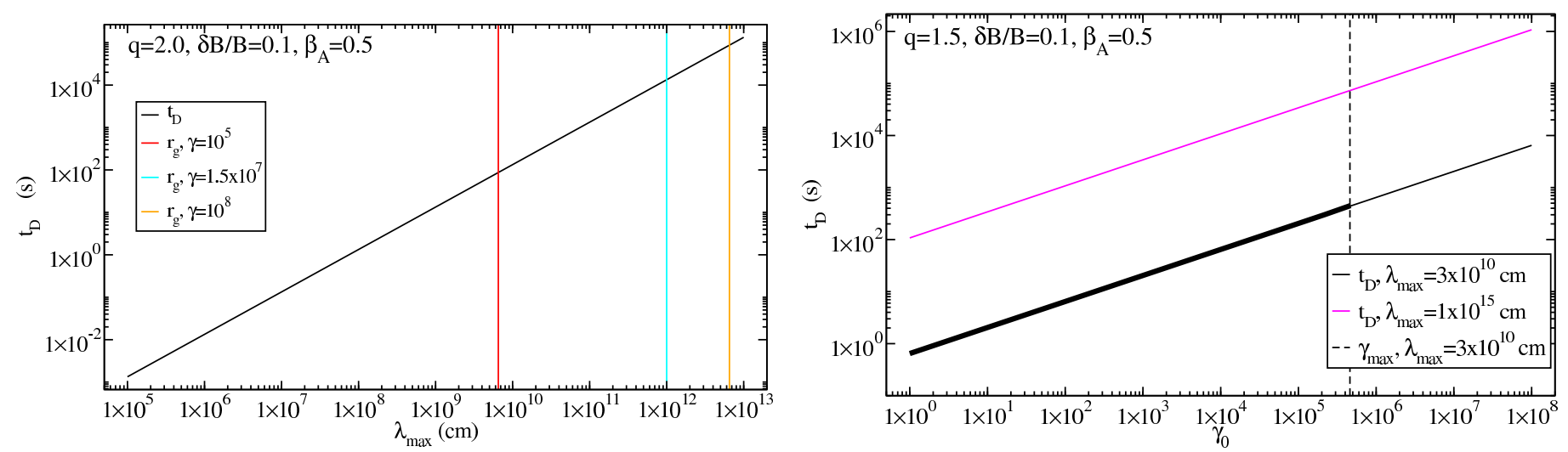

FIG. 3.- Left panel: the $t_{D}$ acceleration time as a function of $\lambda_{\max }$, for $q=2, \delta B / B=0.1$, and $\beta_{A}=0.5$. The vertical lines represent the Larmor radius for $\gamma=10^{5}$ (red line), $\gamma=1.5 \times 10^{7}$ (cyan line), and $\gamma=10^{8}$ (orange line). Right panel: the $t_{D}$ acceleration time for the same parameters as in right panel, for the case of $q=3 / 2$ and as function of $\gamma$, for the two different cases of $\lambda_{\max }=3 \times 10^{10} \mathrm{~cm}$ (black line), and $\lambda_{\max }=1 \times 10^{15} \mathrm{~cm}$ (purple line). The thick black line shows $t_{D}$, for the case of $\lambda_{\max }=3 \times 10^{10} \mathrm{~cm}$, limited to the highest acceleration energy of the particles constrained by the resonant scattering limit: $\rho_{g}=\lambda_{\max }$.
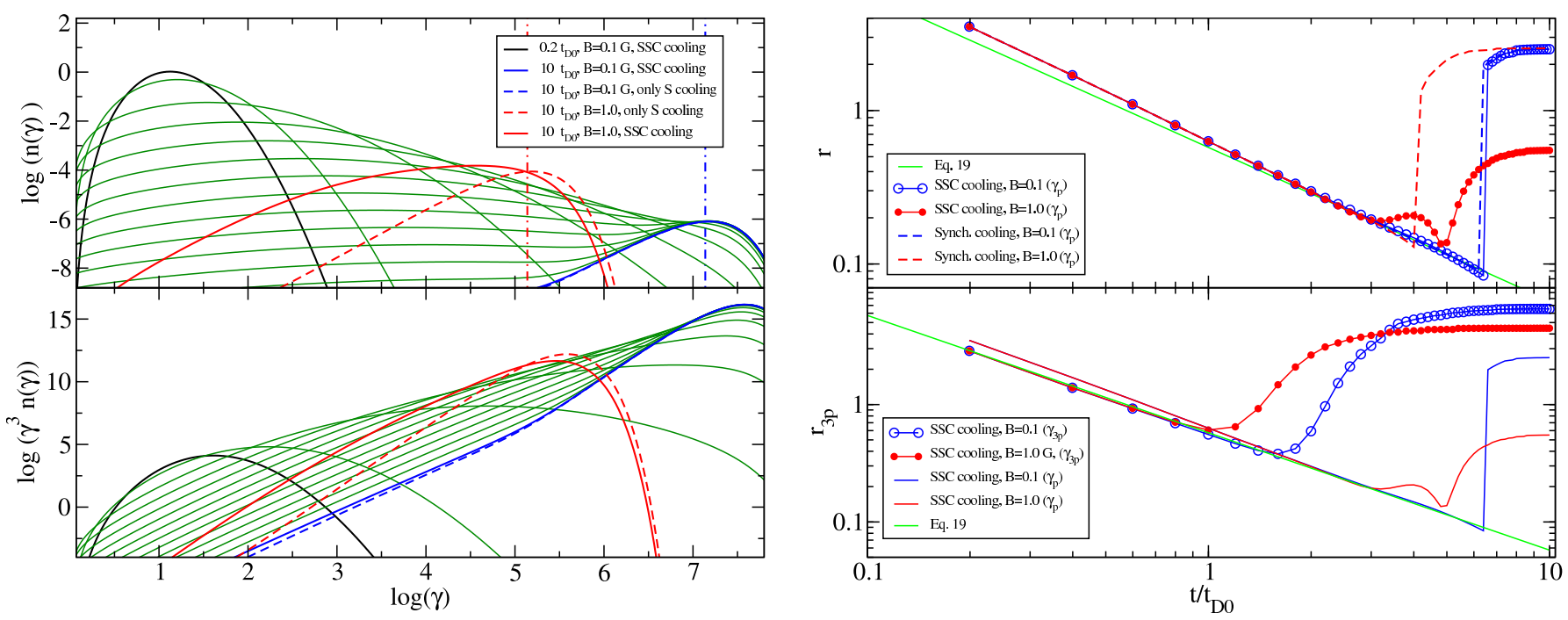

FIG. 4.- Left panels: evolution of the particle spectrum with impulsive injection and no escape for the case of $R=1 \times 10^{15} \mathrm{~cm}$ and $q=2$. Upper panels represent the temporal evolution of $n(\gamma)$, lower panels represent the temporal evolution of $\gamma^{3} n(\gamma)$. Solid lines represent the case of SSC cooling. Red and blue solid lines, represent the final state for $B=1.0 \mathrm{G}$ and $B=0.1 \mathrm{G}$, respectively. Green solid lines represent the temporal evolution, for $B=0.1 \mathrm{G}$, with step of $0.8 \times t_{D}$. The dashed lines represent the final stage in the case of only synchrotron cooling. The vertical dot-dashed lines represent the equilibrium energy in the case on only synchrotron cooling. Right panels: Evolution of the curvature as function of $t / t_{D_{0}}$. Upper panel: curvature $r$ evaluated at $\gamma_{p}$, for the case of SSC cooling (solid red and blue lines) and for the case of only synchrotron cooling (dashed red and blue lines). The solid green line represent the prediction from Eq. 19 Lower panel: the same as in the upper panel, for the curvature $r_{3 p}$ evaluated at $\gamma_{3 p}$ (empty and filled circles) compared to the case of $r$ (solid lines).

particles with larger $\rho_{g}$ (hence larger $\gamma$ ) can't resonate with shorter wavelengths. Taking into account a coherent magnetic field of the order of $0.1 \mathrm{G}$, and $\lambda_{\max } \approx 10^{12} \mathrm{~cm}$ we found that the purely-accelerative efficiency limits the particle energy to $\gamma_{\max } \lesssim 10^{7.5}$. In the left panel of Fig. 3 we plot $t_{D}$, given by Eq. 17] as a function of $\lambda_{\max }$, for the case of $q=2, \delta B / B=0.1$, and $\beta_{A}=0.5$. In this case the acceleration time is energy independent, and for $\lambda_{\max } \approx 10^{12} \mathrm{~cm}$ it will be of the order of $t_{D}=1 / D_{p 0} \approx$ $10^{4} \mathrm{~s}$. In the case of $q \neq 2$, the acceleration will have an energy dependence given by Eq. 17, as shown in the right panel of Fig. 3 for the case of $q=3 / 2$. In this section, we focus on the evolution of the curvature as a function of the momentum-diffusion term, and therefore use only the accelerative contributions coming from the diffusion terms $\left(D_{p}(\gamma), D_{A}(\gamma)\right)$, neglecting the systematic extra term $A(\gamma)$. All the parameters and their numerical values are given in Tab. 1.

\subsection{Impulsive injection}

In the left panels of Fig. 4, and Fig. 5] we plot the evolution of energy distribution $n(\gamma, t)$ (upper panels) and of $\gamma^{3} n(\gamma, t)$ (lower panels) in the case of the impulsive injection without escape, for $q=2$, and for two values of $R: 1 \times 10^{15} \mathrm{~cm}$ (Fig. [4) and $5 \times 10^{13} \mathrm{~cm}$ (Fig. 5). We inject a quasi-monoenergetic electron distribution with $\gamma_{i n j} \approx 10$. The $\gamma^{3} n(\gamma, t)$ representation is useful to compare the results concerning $n(\gamma)$ presented in this section, with those regarding the synchrotron emission presented in Sec. 5. We denote by $\gamma_{p}$ the peak energy of $n(\gamma)$ and by $r$ the curvature evaluated by means of a log-parabolic best fit over a one decade-broad interval centered at $\gamma_{p}$. 

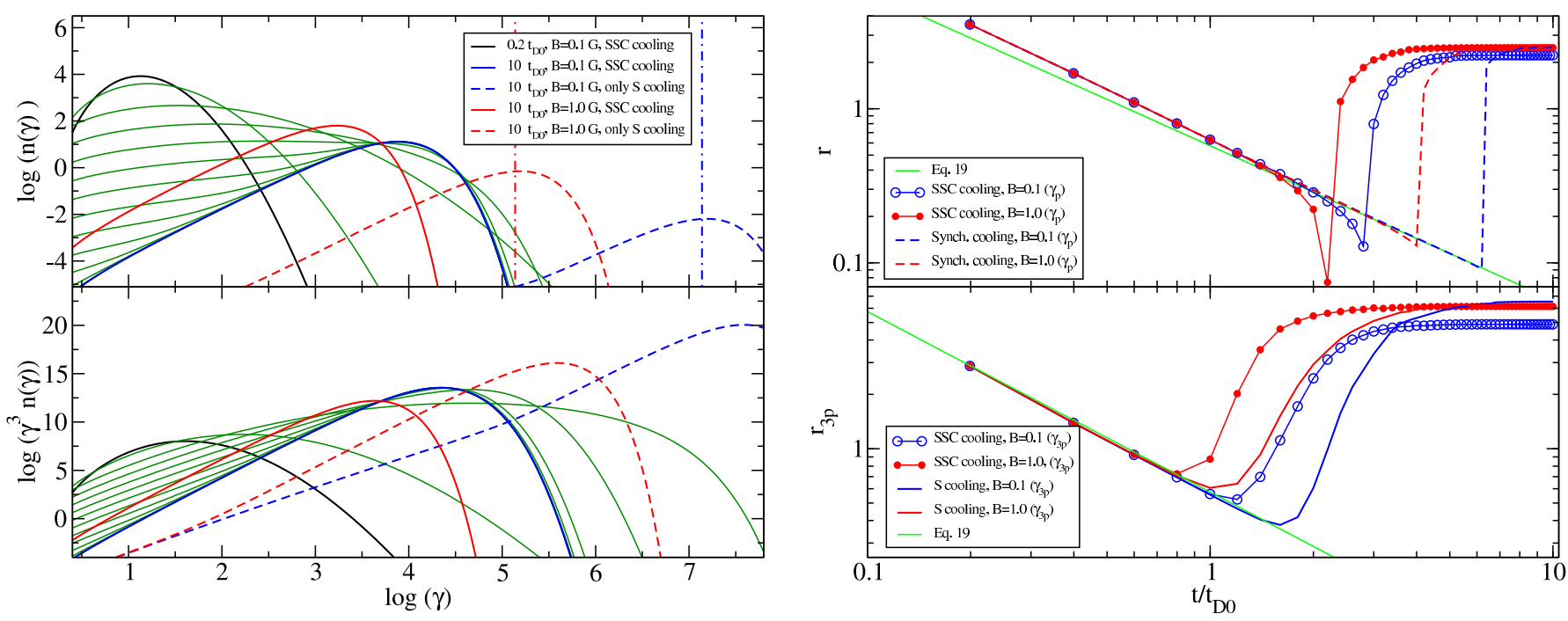

Fig. 5. - Left panels: The same as in Fig. 4 for the case of $R=5 \times 10^{13} \mathrm{~cm}$. Right panels: Upper panel: the same as in Fig. 4 for the case of $R=5 \times 10^{13} \mathrm{~cm}$. Lower panel: the evolution of the curvature $r_{3 p}$, for the case $R=5 \times 10^{13} \mathrm{~cm}$.
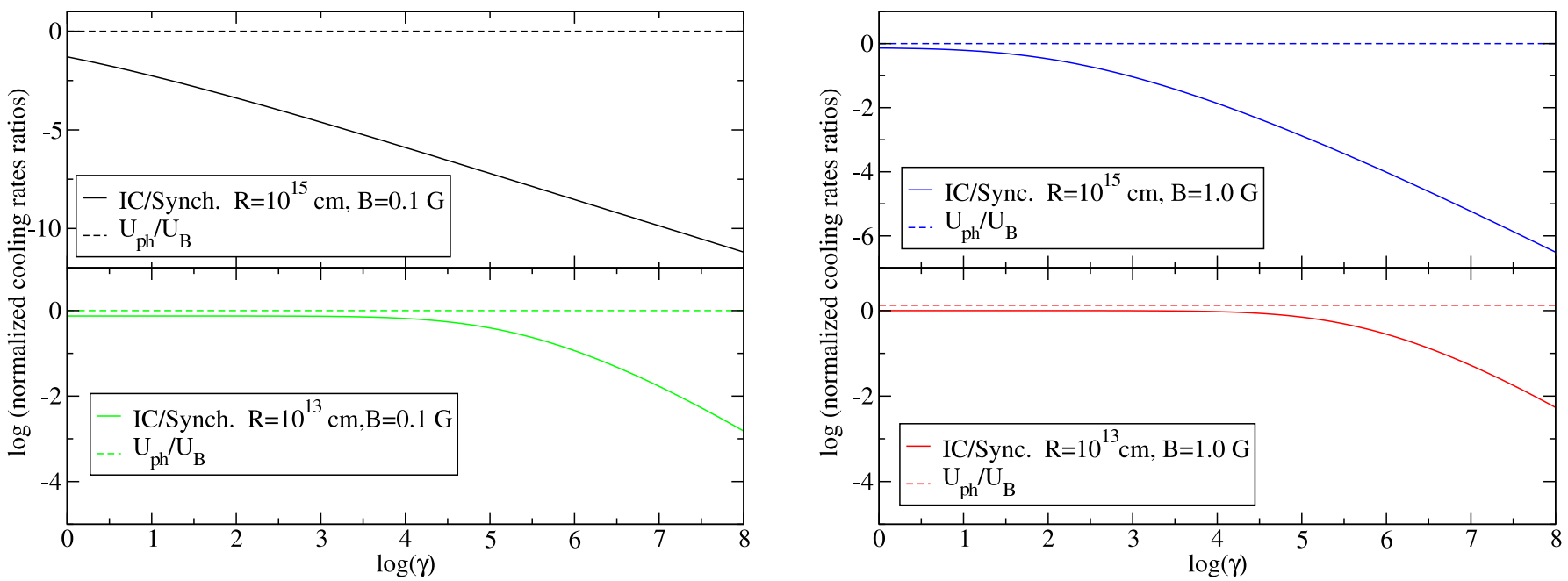

FIG. 6. - Normalized ratios of electron cooling rates $\dot{\gamma}_{I C} / \dot{\gamma}_{S y n c h}$. (solid lines), and $U_{p h} / U_{B}$ (dashed lines), as a function of $\gamma$, for different values of $R$ and $B$, for the case of $q=2$, at the final step of the evolution. Left panels: top, case of $R=10^{15} \mathrm{~cm}$ and $B=0.1 \mathrm{G}$. Bottom, case of $R=10^{13} \mathrm{~cm}$ and $B=0.1 \mathrm{G}$ Right panels: top, case of $R=10^{15} \mathrm{~cm}$ and $B=1.0 \mathrm{G}$, Bottom, case of $R=10^{13} \mathrm{~cm}$ and $B=1.0 \mathrm{G}$

$\gamma_{3 p}$ and $r_{3 p}$ represent the peak of $\gamma^{3} n(\gamma)$ and its curvature, respectively. In the right panels of Fig. 4, and 5, we report on the corresponding temporal evolutions of the curvatures under the effect of both momentum-diffusion and cooling terms. The solid black line corresponds to $t=0.2 \times t_{a c c}$, where $t_{a c c}=t_{D_{0}}$ is the acceleration time due to momentum-diffusion. As the time increases, the diffusion term acts on the distribution by means of both $D_{A}$ and $D_{p}$. The effect of the latter is to make the distribution broader.

One can distinguish three phases: in the first one the energy of particles increases and the curvature parameter decreases following a law $r \propto t^{-1}$ in agreement with the statistical scenario of Sec. 2 and with the Eq. [19] independent of the magnetic field strength $(B=1.0 \mathrm{G}$ and $B=0.1 \mathrm{G}$ ) and of the source size, because the accelerative contribution dominates over the radiative losses; in the second phase, the radiation losses become relevant and the distribution approaches the equilibrium with an increase of the curvature; in the third phase, the balance between acceleration and radiation losses is established and the curvature reaches a stable value.

The equilibrium distribution reached through stochastic acceleration, is described by a relativistic Maxwellian (Stawarz \& Petrosian 2008),

$$
n(\gamma) \propto \gamma^{2} \exp \left[\frac{-1}{f(q, \dot{\gamma})}\left(\frac{\gamma}{\gamma_{e q}}\right)^{f(q, \dot{\gamma})}\right],
$$

where $f(q, \dot{\gamma})$ is a function depending on the exponent of the diffusion coefficient and on the cooling process, and $\gamma_{e q}$ is the Lorentz factor that satisfies the condition $t_{\text {cool }}(\gamma)=t_{a c c}(\gamma)$ and is given by

$$
\gamma_{e q}=\frac{1}{t_{a c c} C_{0}\left(U_{B}+F_{K N}(\gamma)\right)},
$$

with $t_{a c c}$ equal to the fastest acceleration time scale among $t_{A}, t_{D}, t_{D A}$. In the case of Compton dominated cooling we have $\gamma_{e q} \propto \frac{R^{2}}{t_{a c c} B^{2} f_{K N}}$, whilst in the case of 

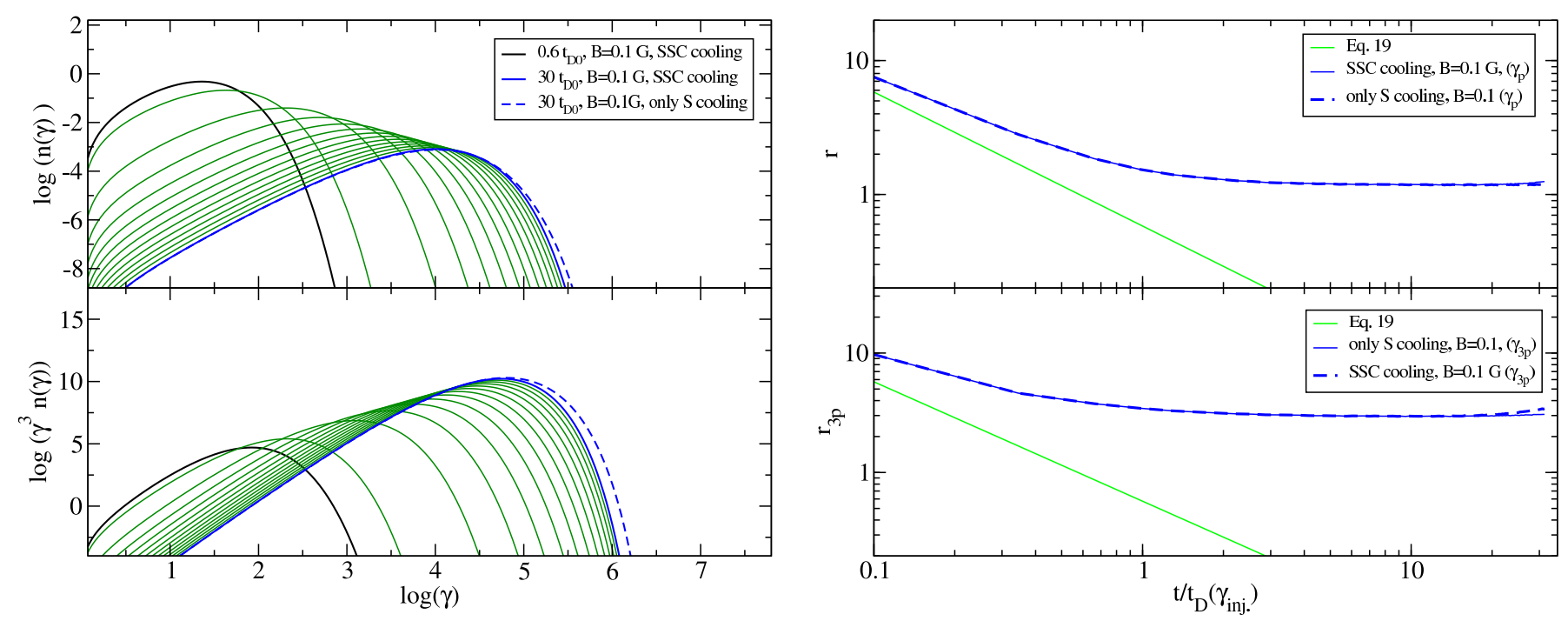

Fig. 7.- Left panes: evolution of the particle spectrum with impulsive injection and no escape for the case of $R=1 \times 10^{15} \mathrm{~cm}, B=1.0$ and $q=3 / 2$. Since $t_{D}$ is energy dependent, on the x-axis we plot the ratio $t / t_{D}\left(\gamma_{i n j}\right)$, where $t_{D}\left(\gamma_{i n j}\right)$ is the diffusive acceleration time evaluated at the injection energy $\gamma_{i n j}$. Green solid lines represent the temporal evolution, for $B=0.1 \mathrm{G}$, with step of $2.4 \times t_{D}\left(\gamma_{0}\right)$. Right panels: Evolution of the curvature $r$ (upper) and $r_{3 p}$ (lower).
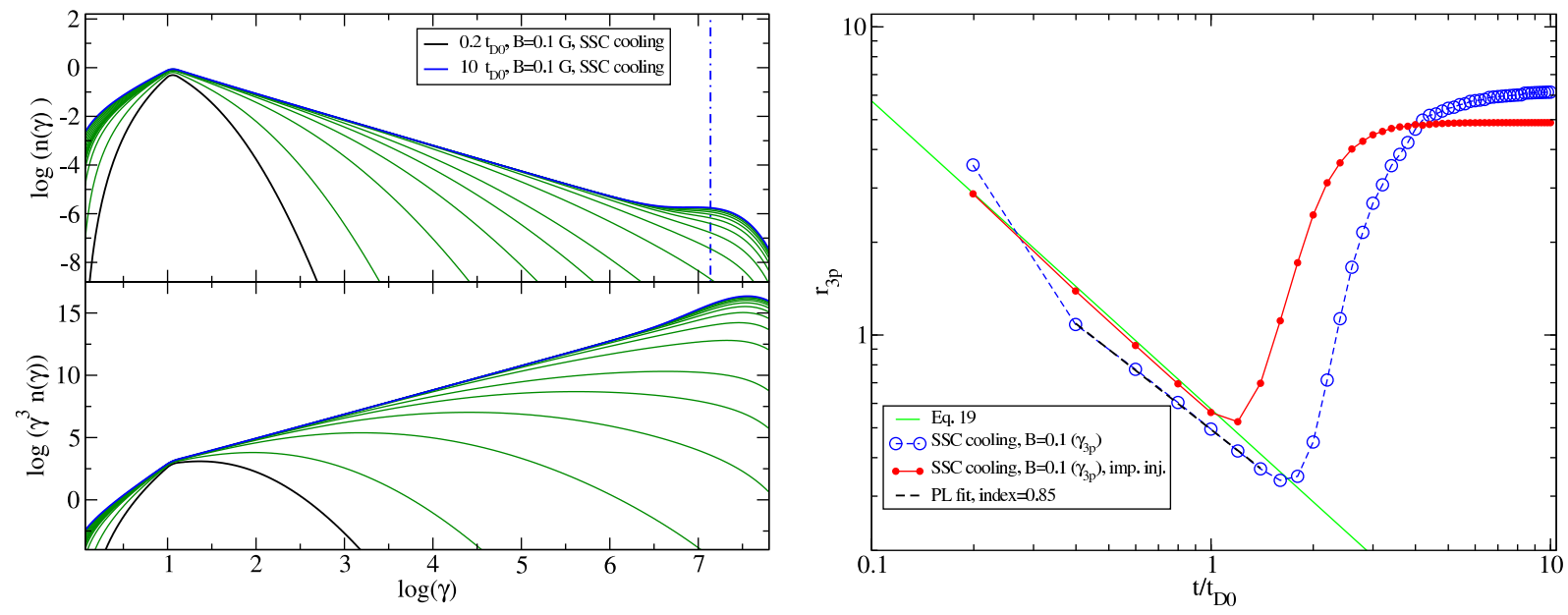

FIG. 8.- Left panel: Evolution of the particle spectrum for continuous injection, $R=1 \times 10^{15} \mathrm{~cm}, B=1.0 \mathrm{G}$, and $q=2$. Lower panel: Evolution of the curvature $r_{3 p}$.

strong KN regime, or in general for synchrotron dominated cooling, we have $\gamma_{e q} \propto \frac{1}{t_{a c c} B^{2}}$. Using a power-law form for the acceleration terms, and in the case of only synchrotron losses (or any cooling process that can be expressed as a power-law function of $\gamma$ ), it is possible to give an analytic expression of $f(q, \dot{\gamma})$ (Katarzyński et al. 2006; Stawarz \& Petrosian 2008). The expectation for synchrotron and IC/TH cooling process, and for $q=2$ is $f(q, \dot{\gamma})=3-q=1$. The curvature resulting from a log-parabolic fit over a decade centered on $\gamma_{p}$ is $r \approx 2.5$, and $r_{3 p} \approx 6.0$ in the case of $\gamma_{3 p}$

We first discuss the case of $R=10^{15} \mathrm{~cm}$ (Fig. (4), with only synchrotron cooling (dashed lines, left panels). In terms of behaviour, we note that for the larger value of $B(1.0 \mathrm{G}$, red lines, right panels), the $r-t$ trend departs from the purely accelerative one $\left(r \propto t^{-1}\right.$, green line right panels) early (relative to the $B=0.1 \mathrm{G}$ case, blue lines in the right panels). This happens because the synchrotron equilibrium energy (vertical dot-dashed lines, left panels) is lower in the case of $B=1.0 \mathrm{G}$.
For both values of $B$, the final values of $r$ are close to the synchrotron equilibrium value of $\approx 2.5$. When IC cooling is also taken into account, the final values of the curvature in $n(\gamma)$ are $r \approx 2.5$ and $r \approx 0.6$, for $B=0.1$ $\mathrm{G}$ and $B=1.0, \mathrm{G}$ respectively. This difference is due to the different IC cooling regimes for the two cases. To show clearly the complexity of the transition from the $\mathrm{TH}$ to the $\mathrm{KN}$ regime, and its dependence on $R$ and $B$, in Fig. 6] we plot the ratio $\dot{\gamma}_{I C} / \dot{\gamma}_{S y n c h}$. (solid lines), and $U_{p h} / U_{B}$ (dashed lines), as a function of $\gamma$ and normalized to unity, for the case of $q=2$, for the final step of the temporal evolution. As long as the ratio $U_{p h} / U_{B}$ is close to $\dot{\gamma}_{I C} / \dot{\gamma}_{S y n c h}$. electrons cool in the full TH regime, and $C(\gamma)=C_{0} \gamma^{2}\left(U_{B}+U_{p h}\right)$. On the contrary, when the electrons radiate in the full $\mathrm{KN}$ regime $\dot{\gamma}_{I C} / \dot{\gamma}_{S y n c h} .<<U_{p h} / U_{B}$. In this case, due to the inefficient KN cooling regime we have $\dot{\gamma}_{\text {Synch. }}>>\dot{\gamma}_{I C}$, and the cooling term is dominated by the synchrotron component: $C(\gamma) \approx C_{0} \gamma^{2} U_{B}$. In the intermediate cases, it's difficult to estimate analytically the ratio $\dot{\gamma}_{I C} / \dot{\gamma}_{S y n c h}$. 
TABLE 1

Parameters values adopted in the numerical solutions OF the diffusion Equation for the CASES Studied in Sec. 4

\begin{tabular}{ll|l|l|l|l}
\hline & & \multicolumn{2}{|c|}{ impulsive inj. } & \multicolumn{2}{c}{ cont. inj. } \\
\hline \hline$R$ & $(\mathrm{~cm})$ & $5 \times 10^{13}, 1 \times 10^{15}$ & - & - & - \\
$B$ & $(\mathrm{G})$ & $0.1,1.0$ & - & - & - \\
$L_{i n j}$ & $(\mathrm{erg} / \mathrm{s})$ & $10^{39}$ & - & $10^{37}$ & - \\
$q$ & & 2 & $3 / 2$ & 2 & $3 / 2$ \\
$t_{D_{0}}=1 / D_{P 0}$ & $(s)$ & $1 \times 10^{4}$ & $1 \times 10^{3}$ & $1 \times 10^{4}$ & $1 \times 10^{3}$ \\
$T_{i n j}$ & $(s)$ & 100 & - & $1 \times 10^{4}$ & - \\
$T_{\text {esc }}$ & $(R / c)$ & $\infty$ & - & 2 & - \\
Duration & $(s)$ & $1 \times 10^{5}$ & - & - & - \\
$\gamma_{\text {inj }}$ & & 10.0 & - & 10.0 & - \\
\hline
\end{tabular}

For $B=1.0 \mathrm{G}$, the $\mathrm{SSC}$ equilibrium is reached at $\gamma \approx$ $3 \times 10^{4}$ and the SSC cooling occurs between the KN and TH regimes (see top-right panel in Fig. 6), hence the value of $f$ is different from unity, as predicted for the case of full IC/TH or synchrotron cooling. When $B=0.1$ $\mathrm{G}$, the equilibrium energy is $\gamma \approx 10^{7}$ and electrons are in extreme KN cooling (see top-left panel in Fig. 6), synchrotron losses are much higher than those due to IC scattering, and again $r$ reaches the previous value of $\approx 2.5$. It is also interesting to note the difference in the trends of $r$ - $t$ and $r_{3 p}-t$. In the latter case, the trend departs form the purely accelerative regime earlier (see Fig 4, right-lower panel) since the electrons with energies close to $\gamma_{3 p}$ are more energetic than those close to $\gamma_{p}$, and thus have much shorter cooling times.

The results for the compact region $\left(R=5 \times 10^{13} \mathrm{~cm}\right)$ are plotted in Fig. 5. Considering that the injected electron luminosity is the same (see Table 1), we expect a different response from the IC cooling, due to the higher photon density $n_{p h}\left(\epsilon_{0}\right)$. The $r$ evolution for the synchrotron cooling case is similar to the previous case, while for the SSC emission, both for the case of $B=1.0 \mathrm{G}$ and $B=0.1 \mathrm{G}$, the final value of $r$ is about 2.5. This is due to the larger photon density which moves the IC scattering into the $\mathrm{TH}$ regime also for the case of $B=0.1 \mathrm{G}$ (compare bottom-left to top-left panel in Fig. 6), hence $n(\gamma)$ approaches the solution of Eq. 26 in the case of $f=1$.

In Fig. 7, we show the temporal evolution for the case of $q=3 / 2\left(R=1.0 \times 10^{15} \mathrm{~cm}, \mathrm{~B}=0.1 \mathrm{G}\right)$. In this case, contrary to the $q=2$ case, the acceleration time $t_{D}$ is energy dependent, hence we study the evolution of $r$ as a function of $t / t_{D}\left(\gamma_{i n j}\right)$, where $t_{D}\left(\gamma_{i n j}\right)$ is the diffusive acceleration time evaluated at the injection energy $\gamma_{i n j}$. The energy dependence of $t_{D}$ affects the evolution of $r$, and the shape of the equilibrium distribution, indeed, the $r-t$, and $r_{3 p}-t$ trends show different behaviour compared to the case of $q=2$. The equilibrium curvature is reached for $t \gtrsim 1 \times t_{D}\left(\gamma_{i} n j\right)$, and the two equilibrium curvature values are $r \approx 1.2$ and $r_{3 p} \approx 3.0$, roughly half of those found for the case of $q=2$, and in agreement with the result from the MC. We note that, the curvature obtained by means of a log-parabolic fit of Eq. 26, for the case $q=3 / 2$ (namely $f=1.5$ ), is $r \approx 3.7$. Hence, both the $\mathrm{MC}$ and the numerical solution of the diffusion equation give a result different from that predicted by the analytical solution in Eq. 26.

\subsection{Continuous Injection}

The case of continuous injection (see Fig. 8) is more complex. The distribution developes a low-energy powerlaw tail, but a log-parabolic bending, driven by the diffusion, is still present at high energies, hence we evaluate the curvature only at $\gamma_{3 p}$, (i.e. the representation useful to compare to the synchrotron SED emission). Spectral curvatures are generally milder than the impulsive injection. In the left panel of Fig. 8 we plot the $r$ - $t$ trend both for the case of impulsive (red lines) and continuous (blue lines) injection, the curvature in the continuous injection case are systematically lower in the pre-equilibrium phases, and in the accelerationdominated stage the trend is again consistent with the "hard-sphere" approximation and statistical approaches. The slope of the electron distribution in the power-law tail is $\approx 1.06$, in good agreement with the predicted one $\approx 1+t_{\text {min-acc }} /\left(2 t_{\text {esc }}\right)=1.075$, consistent with the results of Katarzyński et al. (2006).

\section{EVOLUTION OF THE SPECTRAL PARAMETERS OF SYNCHROTRON AND IC EMISSION}

The most relevant parameters describing the SED of SSC sources provided by observations are the peak energies and curvatures of the synchrotron and IC components. We denote these curvature parameters by $b_{s}$ and $b_{c}$, respectively, and by $E_{s}, E_{c}$ and $S_{s}, S_{c}$, we denote the corresponding SED peak energies, and flux values. We use $\nu_{s}$ and $\nu_{c}$ to indicate the corresponding SED peak frequencies. In the following, we describe the results of the relations between these parameters assuming that electrons are injected into the acceleration region with a quasi mono-energetic spectrum with $\gamma_{i n j} \approx 10$, and using an injection time of $10^{4} \mathrm{~s}$. We use the same working hypothesis for the momentum diffusion coefficient as in Sec. 4.1, and add a systematic acceleration time for the first order process $t_{A}=1.5 \times 10^{3} \mathrm{~s}$, in order to produce $E_{s}$ values ranging between optical and hard X-ray energies. We set the radius of the region at $R=2 \times 10^{15} \mathrm{~cm}$ and the same duration for the injection and acceleration processes, namely $10^{4} \mathrm{~s}$. We varied the other parameters of the model, $B, q$, and $D_{p 0}$ to verify how they affect the relation between the observable ones. All the parameters and their variation ranges are summarised in Tab. 2.

A phenomenological approach, based on the $\delta$ function approximation (Tramacere et al. 2007, 2009; Massaro et al. 2006, 2004), is useful to address the expected relation between the curvature parameters and 

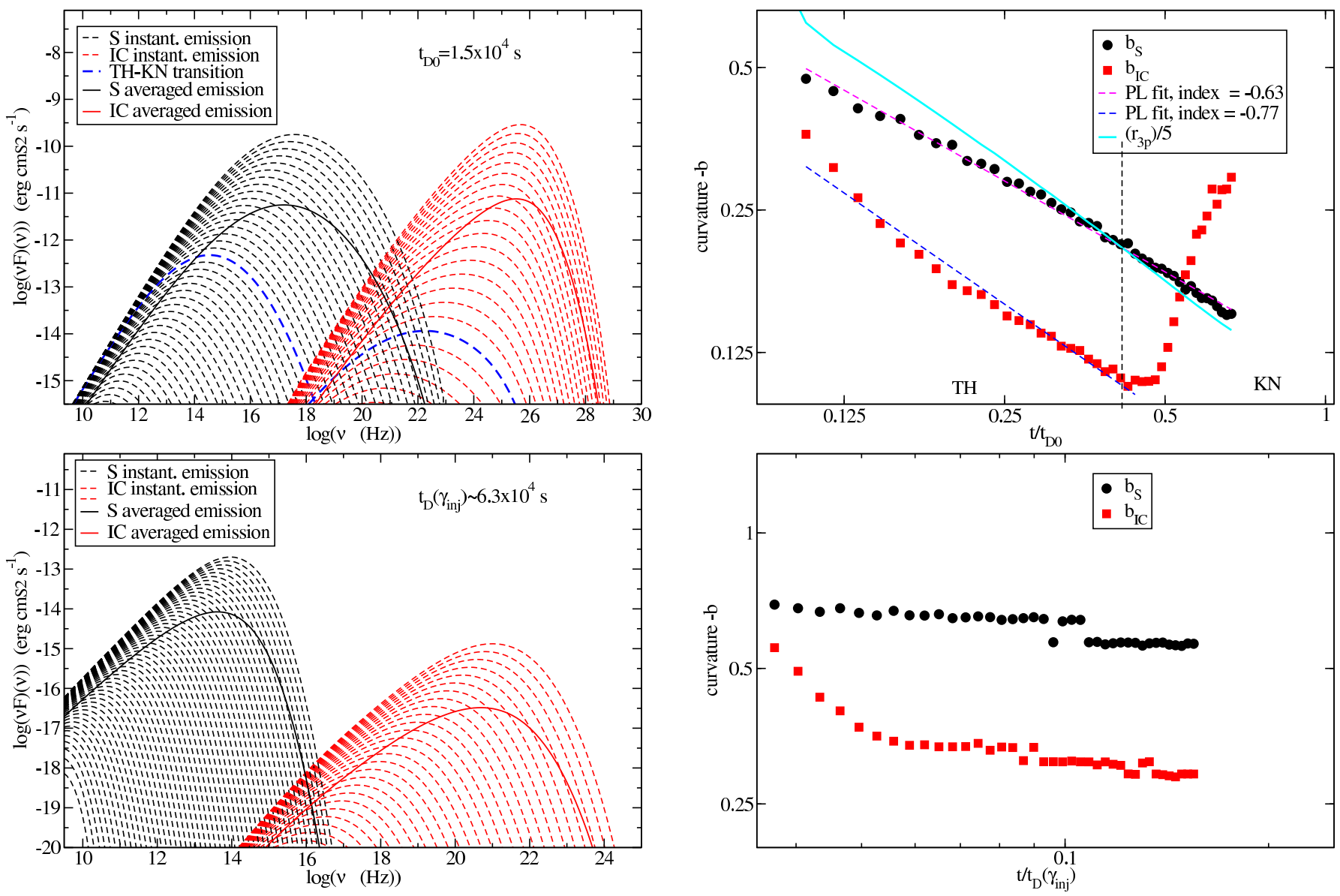

FIG. 9. - Left panel: Evolution of synchrotron (black dashed lines) and IC (red dashed lines) SEDs, for the case of $t_{D_{0}}=1.5 \times 10^{4}$ $\mathrm{s}$ and $q=2$ (top panel), and for the case of $t_{D}\left(\gamma_{i n j}\right) \approx 6.3 \times 10^{4} \mathrm{~s}$ and $q=3 / 2$ (bottom panel). All the other parameters as reported in Tab. 2. The solid lines represent the SEDs averaged overt the full simulation period, and the blue dashed lines (top panel) represent the SEDs corresponding to the transition from TH to KN regime. Right panel: The temporal evolution of $b_{s}$ (black squares) and $b_{c}$ (red squares) as a function of $t / t_{D_{0}}$, for the case of $q=2$ (top panel), and $q=3 / 2$ (bottom panels) The cyan line (top panel) represents the $b_{s}$ trend predicted for the synchrotron emission in case of $\delta$-approximation. The dashed lines (top panel) represent the PL best fit of both $b_{s}$ (purple) and $b_{c}$ (blue) trends.

TABLE 2

PARAMETERS VAlues ADOPTED IN THE NUMERICAL SOlUtions OF THE DIFFUSION EQUATION FOR THE CASES STUdied IN SEC. 5

\begin{tabular}{ll|l}
\hline parameter & & range \\
\hline \hline$R$ & $(\mathrm{~cm})$ & $2 \times 10^{15}$ \\
$B$ & $(\mathrm{G})$ & {$[0.01-1.0]$} \\
$L_{i n j}$ & $(\mathrm{erg} / \mathrm{s})$ & $10^{38}$ \\
$q$ & & {$[3 / 2-2]$} \\
$t_{A}$ & $(\mathrm{~s})$ & $1.8 \times 10^{3}$ \\
$t_{D_{0}}=1 / D_{P 0}$ & $(\mathrm{~s})$ & {$[1.5-25] \times 10^{4}$} \\
$T_{i n j}$ & $(\mathrm{~s})$ & $10^{4}$ \\
$T_{e s c}$ & $(R / c)$ & 2.0 \\
Duration & $(\mathrm{s})$ & $10^{4}$ \\
$\gamma_{i n j}$ & & 10.0 \\
\hline
\end{tabular}

their connections with the peak energies and flux values. According to the standard synchrotron theory (e.g. Rybicki \& Lightman (1986)), in the $\delta$-function approximation, the synchrotron SED peak value and the corresponding peak energy can be expressed by the following relations:

$$
\begin{gathered}
S_{s}\left(E_{s}\right) \propto n\left(\gamma_{3 p}\right) \gamma_{3 p}^{3} B^{2} \delta^{4} \\
E_{s} \propto \gamma_{3 p}^{2} B \delta .
\end{gathered}
$$

which implies

$$
S_{s} \propto\left(E_{s}\right)^{\alpha},
$$

where $\alpha=1.5$ applies for changes of $\gamma_{3 p}$ leaving constant $n\left(\gamma_{3 p}\right), \alpha=2$ for variations of $B$ only, and $\alpha=4$ when the main driver is $\delta$. For a $\log$-parabolic shaped $n(\gamma)$ we have:

$$
\log \left(\gamma_{3 p}\right)=\log \left(\gamma_{p}\right)+\frac{3}{2 r}
$$

and, using the relation $b_{s} \approx r / 5$ (Massaro et al. 2004), or, more precisely, from the analysis presented in Sec. 4.2. $b_{s} \simeq r_{3 p} / 5$. It follows:

$$
\log \left(E_{s}\right) \propto 2 \log \left(\gamma_{p}\right)+\frac{3}{5 b_{s}} .
$$

The relation between $b_{s}$ and $E_{s}$ is:

$$
b_{s}=\frac{a}{\log \left(E_{s} / E_{0}\right)}
$$

with $a=3 / 5=0.6$

The spectral properties of the IC emission are more complex, depending on the transition from the $\mathrm{TH}$ to the KN regime (see Massaro et al. 2006, for a detailed discussion). In the former case, the curvature is close to that of the synchrotron emission, but systematically 
smaller due to the energy redistribution by the scattering process. In the transition to the $\mathrm{KN}$ regime, the energy of IC photons will approach $\gamma m_{e} c^{2}$, hence the IC spectral shape will reflect that of the high-energy tail of $n(\gamma)$, and the curvature $b_{c}$ will be closer to that of the electrons. Then, provided the IC scattering happens in the TH regime, the trends involving $b_{c}$ are expected to be similar to those of $b_{s}$, but showing systematically $b_{c}<b_{s}$. As the KN regime is approached, $b_{c}$ changes differently from $b_{s}$, converging towards $r$.

\subsection{Temporal evolution of $b_{s}$, and $b_{c}$}

We compute the evolution of $b_{s}$ and $b_{c}$, as a function of the time, for the case of $t_{D_{0}}=1.5 \times 10^{4} \mathrm{~s}, B=0.1$ $\mathrm{G}$, and $q=2$, using temporal mesh of $2 \mathrm{~s}$. We plot in the top-left panel of Fig. 9 the instantaneous SEDs at steps of $200 \mathrm{~s}$ : the solid lines represent the synchrotron and IC SEDs averaged over the full duration of the acceleration process $\left(10^{4} \mathrm{~s}\right)$. As the time is increased, the peak energy of both the synchrotron and IC SEDs moves towards higher energies with a broadening of the spectral distribution. The corresponding evolution of curvature parameters is reported in the top-right panel: $b_{s}$ has a trend similar to that of the electron distribution, with $b_{s} \propto\left(t / t_{D 0}\right)^{-\alpha}$, and $\alpha \simeq 0.6$ ( for comparison the cyan solid line represents the $r_{3 p} / 5$ trend, as predicted by the $\mathrm{S} \delta$-approximation). The trend of $b_{c}$, as expected, is more complex because of the transition from TH to KN regime. For $t / t_{a c c} \lesssim 0.4$, it follows the same trend of $b_{s}$ but with systematically lower values. For $t / t_{a c c} \gtrsim 0.4$, when the TH-KN transition occurs, $b_{c}$ increases with time, approaching towards the electron curvature $r$ value. This transition starts for values of $E_{s} \approx 5 \times 10^{-3} \mathrm{keV}$ $\left(\nu_{s} \approx 10^{14} \mathrm{~Hz}\right)$, and $E_{c} \approx 0.05 \mathrm{GeV}\left(\nu_{c} \approx 10^{22} \mathrm{~Hz}\right)$; and the corresponding SEDs are plotted by blue thick-dashed lines in the left panel of Fig. 9.

In the bottom panels of Fig. 9, we show the case of $q=3 / 2$. The synchrotron curvature quickly approaches the equilibrium value of $b_{s} \approx 0.6$, consistent with the equilibrium value $r_{3 p} \approx 3.0$ discussed in Sec. 4.2. In this case we do not observe the $\mathrm{TH} / \mathrm{KN}$ transition in the IC curvature, since the lower values of $E_{s}$ and $E_{c}$, keep the IC scattering mainly in the $\mathrm{TH}$ regime.

\section{2. $E_{s}-S_{s}$ and $E_{s}-b_{s}$ as a function of $D_{p 0}$ and $q$}

The other parameter affecting the evolution of the spectral distributions is the diffusion coefficient $D_{p 0}$ (see Eq. 15) which we assume to vary in the range $\left[1.5 \times 10^{4}, 2.4 \times 10^{5}\right] \mathrm{s}^{-1}$, studying how the main spectral parameters change. In the left top panel of Fig. 10 we plot averaged SEDs for each different value of $D_{p 0}$. The top-right panel shows the trend of $b_{c}$ vs. $D_{p 0}$. As expected, for larger values of $D_{p 0}$, the curvature measured at the peak energy is smaller. The trend is described by a PL with an exponent of about -0.6 for $D_{p 0} \lesssim 2 \times 10^{-5} \mathrm{~s}^{-1}$, and with an exponent of about -0.25 for $D_{p 0} \gtrsim 2 \times 10^{-5} \mathrm{~s}^{-1}$. This break clearly shows the transition between the $\mathrm{TH}$ and $\mathrm{KN}$ regime (marked by a vertical dashed line), indeed it happens for the same values of $D_{p 0}$ corresponding to the $\mathrm{TH} / \mathrm{KN}$ transition in both the $D_{p 0^{-}} b_{c}$ trend, and the $E_{c^{-}} b_{c}$ plot (occurring at $E_{c} \approx 1 \mathrm{GeV}$, see bottom-right panel in Fig. 10). The break in the $D_{p 0}-b_{s}$ trend happens when electrons radiating at $E_{s}$ enter the $\mathrm{KN}$ cooling region, hence, due to the lower cooling level (compared to the TH cooling regime, on the left side of the vertical dashed line), the curvature decreases.

Blue filled circles in the top-left panel represent the peak positions for both SED components. For the synchrotron component, according to Eq. 29, the exponent $\alpha$ in the case of $n\left(\gamma_{3}\right)=$ const, should be 1.5 , while the results of the computations give $\alpha=0.6$. This difference is due to the fact that we inject in the mono-energetic initial distribution, always the same total power that correspond to the same number of particles. When the peak energy increases the distribution becomes broader, implying that the same total number of particles is spread over a larger energy interval and the number of particles contributing to the synchrotron peak emission decreases. Consequently, the $S_{s}-E_{s}$ trend gets softer compared to the predicted value of 1.5 .

We verified quantitatively this effect by computing the trend $n\left(\gamma_{3 p}\right)$ vs $\gamma_{3 p}^{2}$, and found a power-law relation with an exponent of about 0.98 , in nice agreement with the difference between the exponent of 1.5 and that resulting in our simulations. In the bottom panels of Fig. 10 we plot $b_{s}$ vs $E_{s}$ (left), and $b_{c}$ vs $E_{c}$ (right). The $S_{c}-E_{c}$ relation can be fitted by a power-law (orange line, topleft panel in Fig. 10) with the same exponent of the $E_{s}-S_{s}$ relation, as long as the IC scattering, at $E_{c}$ and above, happens in $\mathrm{TH}$ regime. When the KN suppression becomes relevant (green line, top-left panel in Fig. 10), the exponent is larger, and is close to unity.

The synchrotron trend (bottom-left panel in Fig. 10) clearly shows the expected anti-correlation between the peak energy and the spectral curvature, which is well fit by the function given in Eq. 32, with $a=0.68$, not very different from 0.6 , obtained for the $\delta$-function approximation of the synchrotron emission, and assuming that $n(\gamma)$ has a purely log-parabolic shape. A simple powerlaw fit of the same points returns an exponent -0.14 .

We also investigate the role of $q$ on the spectral evolution, setting its variation range to $[3 / 2-2]$, i.e. from the Kraichnan to the "hard-sphere" case. The relations between the spectral parameters are very similar to those found in the previous case with $S_{s} \propto E_{s}^{0.6}$, and $S_{c} \propto E_{c}^{0.9}$. Also in this case, the synchrotron component follows the expectation with a lower curvature for harder turbulence spectra, and the IC trend shows the transition from $\mathrm{TH}$ to KN regime. The power-law best fit of $S\left(E_{s}\right)$ vs $E_{s}$ gives $a=0.88$, larger than that obtained for the case of $D_{p 0}$. In fact, for values of $q$ lower than 2, corresponding to less turbulence and hence diffusion, the curvature gets higher values and the peak energy lower values, compared to the "hard-sphere" case. The power-law fit for $b_{s}$ vs $E_{s}$ returns an exponent of -0.16 , practically coincident with the previous one, indicating that the average properties of these parameters are the same in both the $q$ and $D_{p 0}$ cases.

$$
\text { 5.3. } E_{s, c}-S_{s, c} \text { and } E_{s, c}-b_{s, c} \text { as a function of } B
$$

The magnetic field $B$ drives the radiative losses, which affect the evolution of the spectral parameters. In Sec. 4. we showed that different cooling conditions, and the transition from $\mathrm{TH}$ to $\mathrm{KN}$, can determine very different values of $\gamma_{e q}$, for the same acceleration conditions. Assuming that the acceleration time scale is independent of the magnetic field, Eq. 27 shows that $\gamma_{e q} \propto 1 / B^{2}$, 

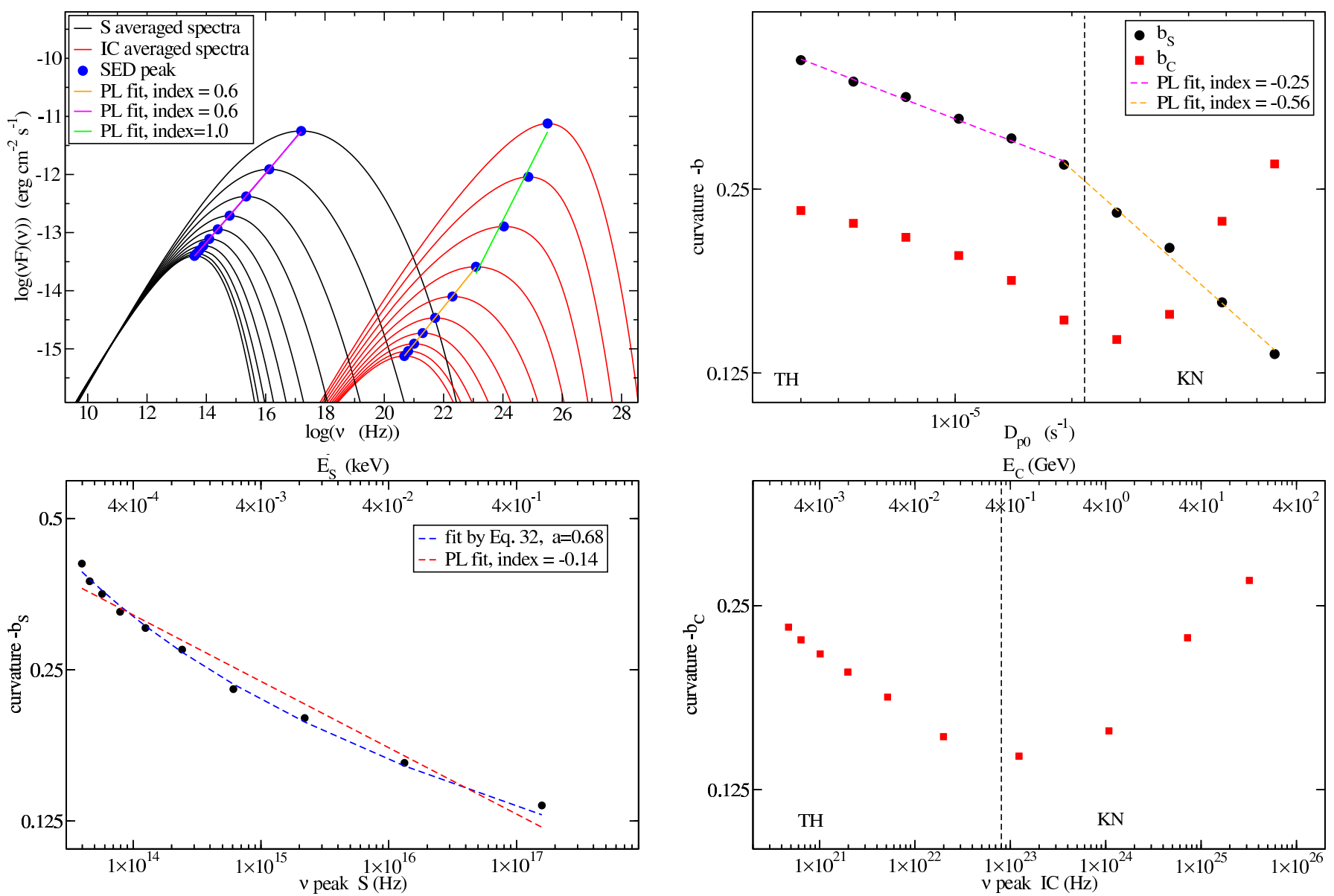

FIG. 10.- Upper left panels: synchrotron (red lines), and IC (red lines) average SEDs for each different value of $t_{D_{0}}$ in the range reported in Tab. 2, with $q=2$. Blue points represent the position of $E_{S, C}$ and $S_{S, C}$. The purple, orange, and green line represent the PL best fit of the $E_{S}-S_{S}$ and $E_{C}-S_{C}$ trends. Upper right panel: $b_{s}$ and $b_{c}$, for each average SED in the right panel, as a function of $D_{p 0}$. Dashed lines represent the PL best fit of the $b-D_{p 0}$ trend. Lower left panel: the $b_{s}-E_{s}$ trend obtained by means of a log-parabolic best fit of the averaged SEDs plotted in the upper right panel Lower left panel: same as in the lower left panel, for the $b_{c}-E_{c}$

implying that, as long as $B$ is small enough to result in $\gamma_{3 p}<<\gamma_{e q}$, the evolution of $n(\gamma)$ around the peak value is dominated by the acceleration terms, while, for values of $B$ resulting in $\gamma_{3 p} \gtrsim \gamma_{e q}$ the evolution obtains a notable contribution due to cooling. In the top-left panel of Fig. 11, we plot the averaged SEDs. According to Eq. 28, and 29, the synchrotron peak value should scale as $S_{s} \propto\left(E_{s}\right)^{2}$. Indeed, for values of $B \lesssim 0.2 \mathrm{G}$ we obtain an exponent equal to 2.04 , very close to the value found with the $\delta$ - approximation. For higher values of the magnetic field, $E_{s}$ is anti-correlated with $S_{s}$. This behaviour represents a cooling signature due to the decreasing value $\gamma_{e q}$ for increasing $B$ values, with $\gamma_{e q}$ getting closer to $\gamma_{3 p}$. This is confirmed both by the shape of the synchrotron SEDs and by the $b_{s}-B$ plot (top-right panel in Fig. 11). Indeed, $\mathrm{S}$ SEDs for $B \gtrsim 0.2 \mathrm{G}$, exhibit an exponential decay, meaning that the distributions have reached, or are close to reaching, the equilibrium energy. Consistently with the $\mathrm{S}$ shape evolution, the $b_{s}-B$ relation shows an almost stable value of $b_{s}$ for $B \lesssim 0.2 \mathrm{G}$, and an increasing trend for $B \gtrsim 0.2 \mathrm{G}$. This change, in both the $S_{s}-E_{s}$ and $b_{s}-B$ trends, is interesting and can provide a useful phenomenological tool to understand the evolution of non-thermal sources. Another interesting feature is shown in the $S_{c}-E_{c}$ plot: for $B \lesssim 0.2 \mathrm{G}$ the IC peak energy is practically constant, as expected in the KN limit from the kinematical limit relating the scattered photons energy to that of the electrons: $h \nu_{I C} \approx \gamma m_{e} c^{2}$. In fact, photons at energies $\approx E_{c}$ are produced in the KN regime, and for $B \lesssim 0.2 \mathrm{G}$ the electron peak energy $\gamma_{3 p}$ is constant, so $E_{c}$ must also be constant. For $B \gtrsim 0.2$ $\mathrm{G}, \gamma_{3 p}$ decreases because of cooling, and, accordingly, $E_{c}$ also decreases. This is another interesting test that can provide a probe for $B$ driven flares evolving to the KN regime. The $E_{s}-b_{s}$ plot in the bottom-left panel of Fig 11. confirms the cooling signature discussed above, showing $b_{s}$ uncorrelated with $E_{s}$ as long as $\gamma_{3 p}<<\gamma_{e q}$, and an increasing value of $b_{s}$ with $E_{s}$ almost stable, when $\gamma_{3 p} \gtrsim \gamma_{e q}$

\section{SPECTRAL EVOLUTION OF HIGH ENERGY FLARES OF BRIGHT HBL OBJECTS}

The previous considerations on the spectral evolution of SSC sources, in which high energy electrons are accelerated in a relatively short timescale by stochastic processes, can be successfully applied to describe the behaviour of some bright HBLs objects. These sources are, in fact, characterised by having the synchrotron peak in the $\mathrm{UV} / \mathrm{X}$-ray range and the $\mathrm{IC}$ peak in $\gamma$ rays up to $\mathrm{TeV}$ energies. Several flares, observed simultaneously in both these ranges, exhibited SEDs very well described by a log-parabolic law, whose parameters, particularly their 

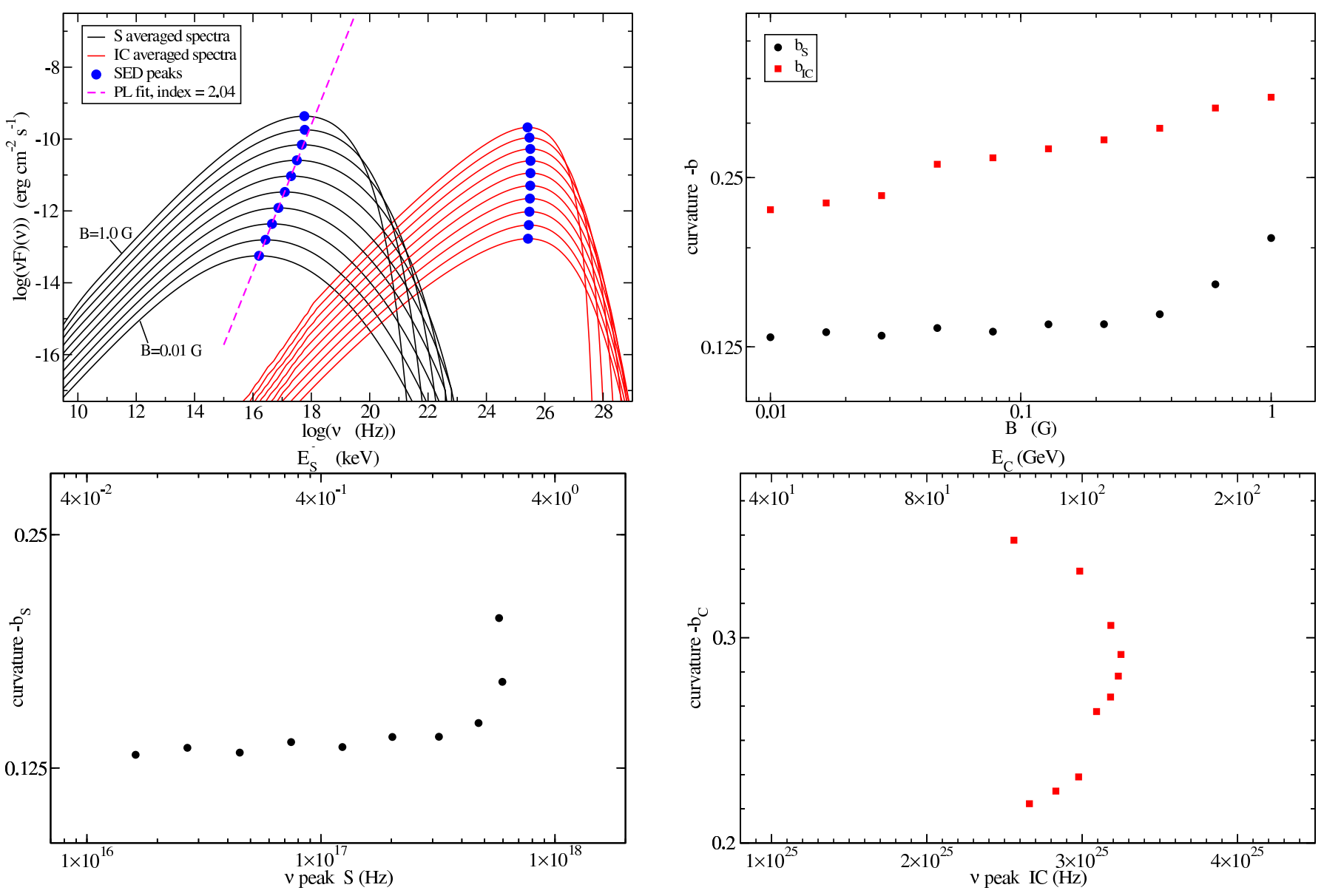

FIG. 11.- Same as in Fig. 10 for different values of $B$ in the range reported in Tab. 2.

curvature, are estimated with high accuracy. A similar analysis for low-energy peaked BL Lac objects is much more difficult because the peak of their synchrotron component is typically in the infrared range and the available simultaneous multifrequency data are extremely few. Tramacere et al. (2007, 2009) and Tramacere (2007) pointed out that the observed anticorrelation between $E_{s}$ and $b_{s}$ in the synchrotron SED of Mkr 421, can provide a clear signature of a stochastic component in the acceleration process. In the same analysis, these authors presented also an interesting correlation between $E_{s}$ and $S_{s}$. Massaro et al. (2008) found that the $E_{s^{-}} b_{s}$ and $E_{s^{-}}$ $S_{s}$ trends hold also for a larger sample of eleven HBLs, making stronger the hypothesis that a common accelerative mechanism may drive such physical processes for this class of active galactic nuclei . To give a theoretical framework to these phenomenological relations, we try to reproduce both the $E_{s}-b_{s}$ and $E_{s}-S_{s}$ relations derived from the data of the aforementioned papers. In the following we will consider the data of Mrk 421 from Tramacere et al. (2007, 2009), collected over a period of 13 years, and of six HBL objects from Massaro et al. (2008): Mrk 180, Mrk 501, PKS 0548-322, PKS 1959$650,1 \mathrm{H} 1426+428$, covering a period of about 11 years and including both quiescent and flaring states. The sources from Massaro et al. (2008) were chosen because the data are good enough to safely constrain both curvature and $E_{s}$ values, and because the observed variations of the sample luminosity are compatible with the assumption to be driven by changes of $E_{s}$.

Following the analysis presented in Sec. 5] we consider two scenarios in which these trends are driven by the momentum-diffusion term. In the first case, the momentum diffusion changes because of variations of $D_{p 0}$, due to changes of $\delta B / B$ or $\beta_{A}$, but the turbulence spectrum $(q=2)$ remains stable. In the second scenario the turbulence spectrum is variable with $q$ ranging in $[3 / 2,2]$. We use the same method described in Sect. 5. to compute the averaged SEDs for each value of $D_{p}$ (or $\left.q\right)$; computations are performed for three values of the magnetic field $\mathrm{B}=0.05,0.1$, and $0.2 \mathrm{G}$. All the model parameters are summarised in Tab. 3.

The comparison with the data can be affected by an observational bias due to the limited energy range of detectors. In fact, when the peak energy is close to the limits the curvature is not well estimated because one can use only a portion of the parabola below or above the peak. Generally, curvatures lower than the actual ones are obtained. The energy range $[0.5,100.0] \mathrm{keV}$ is the typical spectral window covered by X-ray and hard-Xray detectors. In our analysis we used this fixed window to take into account this possible bias in the observed data when $E_{s}$ is variable.

\section{1. $E_{s}-b_{s}$ relation}

The $E_{s}-b_{s}$ trend, and in particular the anticorrelation between these two observables parameters, is the strongest signature of a stochastic component in the ac- 

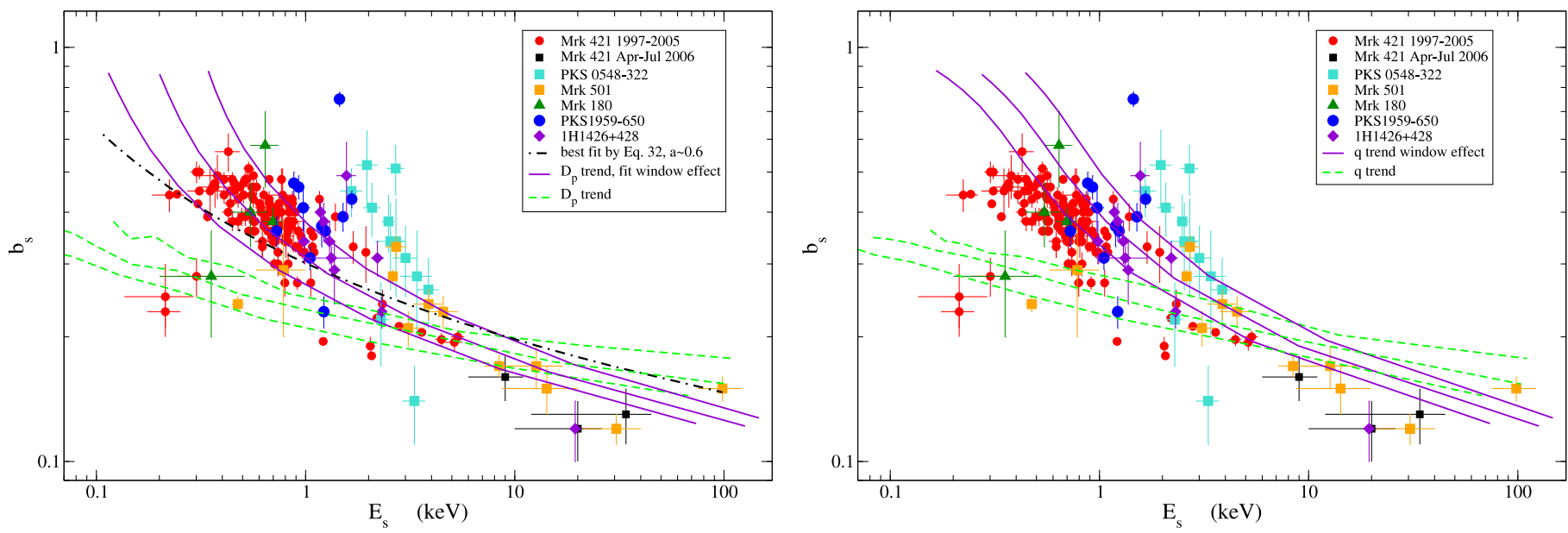

FIG. 12.- Left panel: the $E_{s}-b_{s}$ trend observed for the six HBLs in our sample. The dashed green lines represent the trend reproduced by stochastic acceleration model, for the parameters reported in Tab. 3 , and for the $D$ trend, the different lines corresponding to three different values of $B$ reported in Tab. 3. The purple lines represent the trend obtained by fitting the numerically computed SED over a fixed spectral window in the range $0.5-100 \mathrm{keV}$. Right panel: the same as in the left panel for the case of the $q$ trend.

TABLE 3

PARAMETERS' VAlues ADOPTED IN THE NUMERICAL SOlUtions OF THE DIFFUSION EQUATION TO REPRODUCE THE OBSERVED TRENDS of THE HBLs Reported IN SEC. 6

\begin{tabular}{ll|l|l}
\hline & & $D$ trend & $q$ trend \\
\hline \hline$R$ & $(\mathrm{~cm})$ & $3 \times 10^{15}$ & - \\
$B$ & $(\mathrm{G})$ & {$[0.05-0.2]$} & - \\
$L_{i n j}\left(E_{s}-b_{s}\right.$ trend $)$ & $(\mathrm{erg} / \mathrm{s})$ & $5 \times 10^{39}$ & - \\
$L_{\text {inj }}\left(E_{s}-L_{s}\right.$ trend $)$ & $(\mathrm{erg} / \mathrm{s})$ & $5 \times 10^{38}, 5 \times 10^{39}$ & - \\
$q$ & & 2 & {$[3 / 2-2]$} \\
$t_{A}$ & $(\mathrm{~s})$ & $1.2 \times 10^{3}$ & - \\
$t_{D_{0}}=1 / D_{P 0}$ & $(s)$ & {$\left[1.5 \times 10^{4}-1.5 \times 10^{5}\right]$} & $1.5 \times 10^{4}$ \\
$T_{i n j}$ & $(s)$ & $10^{4}$ & - \\
$T_{\text {esc }}$ & $(R / c)$ & 2.0 & - \\
Duration & $(s)$ & $10^{4}$ & - \\
$\gamma_{\text {inj }}$ & & 10.0 & - \\
\hline
\end{tabular}

\section{celeration.}

In Fig. 12 we report the scatter plot in the $E_{s}-b_{s}$ plane for the six considered sources. The left panel reports the results obtained by changing the value of $D_{p 0}$ : the green dashed lines describe the trend resulting from a log-parabolic fit of the synchrotron SED over a decade in energy centered on $E_{s}$; the purple lines represent the same trend obtained by fitting log-parabola in the fixed spectral window $[0.5,100.0] \mathrm{keV}$. Both these trends are compatible with the data and track the predicted anticorrelation between $E_{s}$ and $b_{s}$. Purple data, however, give a better description, hinting that the "window" effect could be a real bias. Each of the three lines was computed for a different value of the magnetic field. It is remarkable that the variation of a single parameter, $D_{p 0}$ can describe the observed behaviour. The dispersion in the data is relevant, and can be related to the variation of $B$ (as partially recovered by numerical computation), or by different values of the beaming factor, $R$, and $L_{i n j}$, during different flares, and for different objects.

The dot-dashed tick line represents the best fit of the observed data by means of Eq. 32. and returns a value of $a \approx 0.6$, as expected from theoretical predictions for the case of the $\delta$-approximation, and pure log-parabolic electron distribution. This fitted line is also compatible with the numerical trend shown by the purple lines. Note that the observed curvature values are in the range $[0.1$, $0.5]$, corresponding to $r_{3 p} \sim[0.5,3.0]$. According to the results presented in Sec. 4.2. the expected equilibrium curvature in the synchrotron emission, in the full $\mathrm{KN}$ or TH regime, and for $q=2$, should be of $r_{3 p} \approx 6.0$, and of $r_{3 p} \approx 5.0$ in the intermediate regime. In the case of $q=3 / 2$, the equilibrium curvature should be $r_{3 p} \sim 3.0$. This is perhaps an interesting hint that, both in the flaring and the quiescent states, for $q=2$, the distribution is always far from equilibrium. In the case of $q=3 / 2$, only for $E_{s} \lesssim 1.5 \mathrm{keV}$ the curvature are compatible with the equilibrium $\left(r_{3 p} \simeq 3.0\right.$, corresponding to $\left.b_{s} \sim 0.6\right)$. For larger values of $E_{s}$, we find again curvature well below the equilibrium value. These results provide a good constraint on the values of the magnetic field $B \lesssim 0.1 \mathrm{G}$.

The $q$-driven trend (right panel) is also compatible with the data, but for values of $E_{s} \lesssim 1 \mathrm{keV}$, the $D_{p 0^{-}}$ driven case seems to describe better the observed behaviour, but any firm conclusion is not possible because of the dispersion of the data.

\section{2. $E_{s}-L_{s}$ trend}

As a last benchmark for the stochastic acceleration model, we reproduce the observed correlation between $E_{s}$ and $S_{s}$, which follows naturally from the variations of $D_{p 0}$ and $q$. Considering that the redshifts of the six considered HBL objects are different, we prefer to use their peak luminosity $L_{s}=S_{s} 4 \pi D_{L}^{2}$, where $D_{L}$ is the luminosity distance ${ }^{1}$. To account for the different jet power of sources, we considered two data subsets, and we assumed $L_{i n j}=5 \times 10^{39} \mathrm{erg} / \mathrm{s}$ for the first subset (top panels of Fig. 13), and $L_{i n j}=5 \times 10^{38}$ for second (bottom panels of Fig. 13). In the left panels of Fig. 13. we report the $D_{p 0}$ driven trend, and in the right panels the $q$ driven trend. Solid lines represent the trend obtained by deriving $L_{s}$ from the log-parabolic best fit of the numerically computed SEDs, centered on $E_{s}$; dashed lines are the trends obtained by fitting the numerical results in the fixed energy window $[0.5,100] \mathrm{keV}$.

1 We used a flat cosmology model with: $H_{0}=73$ $\mathrm{km} / \mathrm{s} / \mathrm{Mpc}, \Omega_{\text {matter }}=0.27, \Omega_{\text {vacuum }}=0.73$ 

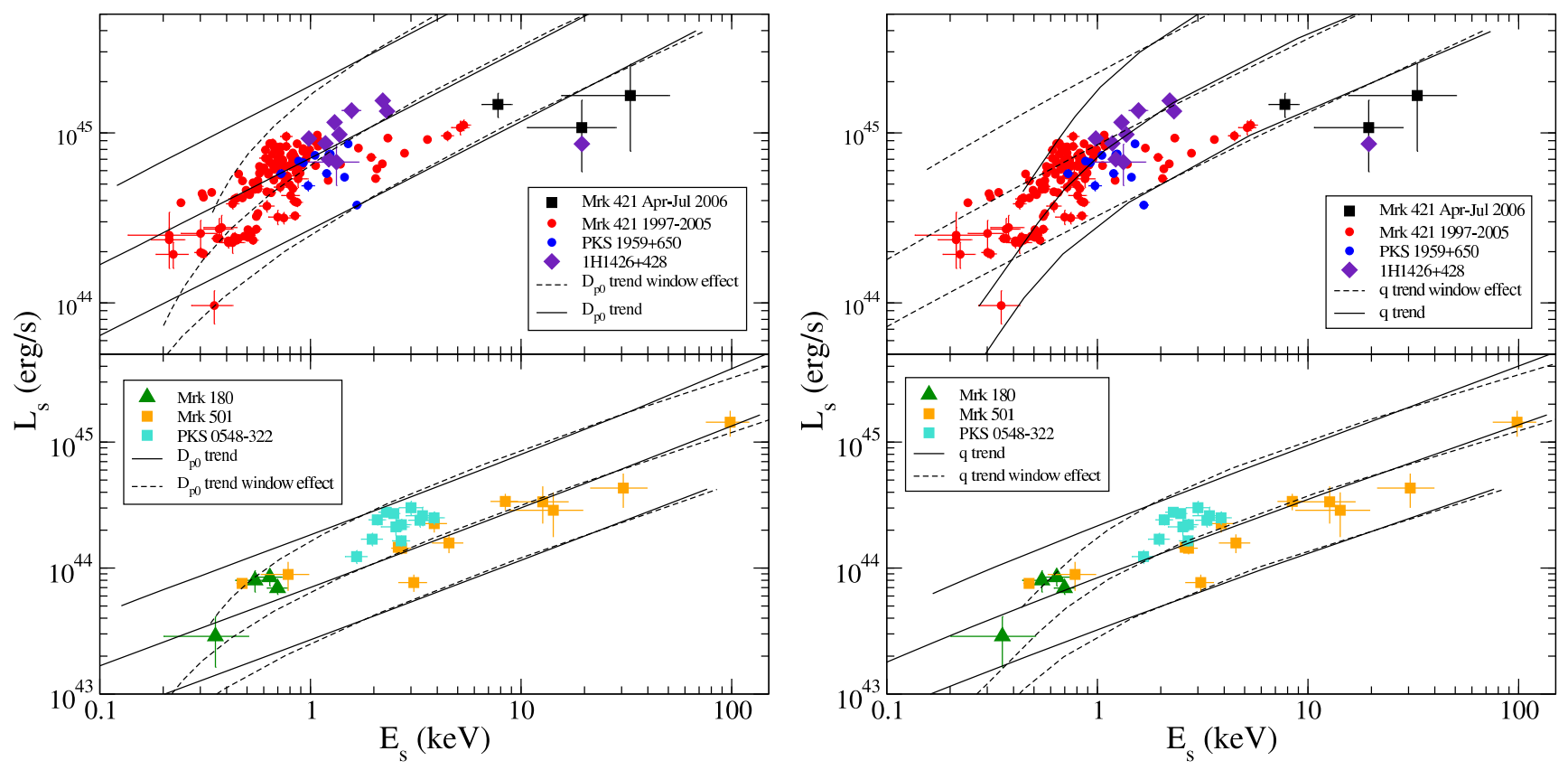

Fig. 13. - Left panels: the $E_{s}-L_{s}$ trend observed for six HBLs in our sample, top panel corresponds to the case of $L_{i n j}=5 \times 10^{39} \mathrm{erg} / \mathrm{s}$, bottom panel corresponds to the case of $L_{i n j}=5 \times 10^{38}$. The solid black lines represent the trend reproduced by stochastic acceleration model, for the parameters reported in Tab. 3 , and for the $D$ trend, the different lines corresponding to three different values of $B$ reported in Tab. 3. The dashed lines represent the trend obtained by fitting the numerically computed SED over a fixed spectral window in the range $0.5-100 \mathrm{keV}$. Right panels: the same as in the left panel for the case of the $q$ trend.

Both the results give a good description of the observed data, and their shapes are similar. Solid lines follow well a power-law with an exponent of about 0.6 , while the windowed trends (dashed lines) show a break around 1 $\mathrm{keV}$ and the exponent below this energy turns to about 1.5. A similar break at the same energy, can be noticed in the points of Mrk 421 in the $E_{s}-S_{s}$ plot presented by Tramacere et al. (2009), who found an exponent of $\sim 1.1$ and of $\sim 0.4$ below and above $1 \mathrm{keV}$, respectively. This could again be an indication that the observed values are actually affected by the bias.

\section{DISCUSSION}

Broad band observations of non-thermal sources have shown that the spectral curvature at the peaks of their SEDs can now be measured with good accuracy. In this paper, we have presented, using different approaches, the relevance of these data for the understanding of the competition between statistical acceleration and radiation losses. First, using a simple statistical approach and Monte Carlo calculations, we have shown that the logparabolic energy distribution of the relativistic electron is a good picture in the first phases before equilibrium is reached. In this case the curvature decreases with time and, therefore, for increasing peak energies. This evolution is confirmed by numerical solutions of the diffusion equation taking properly into account both stochastich acceleration and radiative SSC cooling. The major results can be summarised as follows.

The evolution of the electron energy distributions (Sec. 4) shows that:

- in the case of synchrotron and SSC cooling, and for all the values of $B$ and $R$, as long as the distribution is far from equilibrium, the trend on $r$ is dictated by $D_{p}$, and is well described by Eq. 19 .
- when the distributions approach equilibrium, the value of $r$ is determined by the shape of the equilibrium distribution, which is a relativistic Maxwellian, with the sharpness of the cuf-off determined by both $q$ and the IC cooling regime;

- in the case of $q=2$, and for equilibrium energies implying that IC cooling happens either in the TH regime or in the extreme $\mathrm{KN}$ regime (IC cooling negligible compared to the synchrotron one), the numerical solution of the diffusion equation follows the analytical prediction $(f=1$, that holds for any $\dot{\gamma} \propto \gamma^{2}$ ), and the corresponding equilibrium curvature is $r_{3 p} \approx 6.0\left(b_{s} \approx 1.2\right)$. In the case of $q=3 / 2$ the equilibrium curvature is $r_{3 p} \approx 3.0\left(b_{s} \approx 0.6\right)$. These limiting values could be a useful observational test to find cooling dominated flares with the distribution approaching to the equilibrium;

- when cooling is in the intermediate regime between TH and KN and for the $q=2$ case, the condition $f=1$ fails, and the end values of $r$ decrease, strongly depending on the balance between $U_{B}$ and the seed IC photon energy $\left(U_{p h}\right)$; numerical computations are necessary to evaluate the right value of $r$ at equilibrium.

The analysis of the spectral evolution of SSC emission (Sec 5) shows that:

- changes of $D_{p 0}$ (or $q$ ) imply that the curvature and peak energy of the synchrotron emission are anticorrelated; the $E_{s}-b_{s}$ trend can be phenomenologically described by Eq. 32 ,

- The $E_{c}-b_{c}$ trend presents a clear signature of the transition from the $\mathrm{TH}$ to the $\mathrm{KN}$ regime. In particular when the IC scattering approaches the KN 
regime we observe a sharp change in the $b_{c}$, with a positive correlation with $E_{c}$, whilst in the $\mathrm{TH}$ regime the correlation is negative as in the case of the $E_{s}-b_{c}$;

- the magnetic field plays a relevant role on the cooling process, and $B$ driven variations present relevant differences compared to those due to $D_{p 0}$ (and q).

In particular, for what concerns the $\mathrm{B}$ driven case, we note first that the $E_{s}-S_{s}$ correlation follows the prediction of the synchrotron theory and shows the power-law relationship with $E_{s} \propto\left(S_{s}\right)^{\sim 2.0}$. On the contrary, in the case of $D_{p 0}$ and $q$ changes, we find $E_{s} \propto\left(S_{s}\right)^{0.6}$. Another relevant difference in the $B$ driven case is the evolution of $S_{c}$. For the case of $D_{p 0}$ and $q$ driven trends $S_{c}$ relates to $E_{c}$ through a power-law with exponent of about [0.7-0.8]. On the contrary, for the $B$ driven case with IC scattering in the full $\mathrm{KN}$ regime, the value of $E_{c}$ is almost constant and uncorrelated with $S_{c}$ (see Fig. 11), due to the kinematic limit of the KN regime. $E_{c}$ starts to decrease when $B$ is enough large to make dominant the cooling process. This is an interesting signature that could be easily checked in the observed data.

The comparison of the $E_{s}-b_{s}$ and $E_{s}-S_{s}$ trends, ob- tained through several X-ray observations of six HBL objects spanning a period of many years, with those predicted by the stochastic acceleration model, shows very good agreement. We are able to reproduce these longterm behaviours, by changing the value of only one parameter $\left(D_{p 0}\right.$ or $\left.q\right)$. Interestingly, the $E_{s}-S_{s}$ relation follows naturally from that between $E_{s}$ and $b_{s}$. This result is quite robust and hints at a common accelerative scenario acting in the jets of HBLs.

As a last remark, we note that very recently Massaro \& Grindlay (2011) find also in the case of GRBs a $E_{s}-b_{s}$ trend, similar to that observed in the case of HBL objects. They measured values of the curvature up to 1.0, typically higher than in HBLs. It's interesting to note that the value of 1.0 is close to the limit of $\sim 1.2$, that we predict in the case of distributions approaching the equilibrium in either $\mathrm{TH}$ or $\mathrm{KN}$ regime, for $q=2$.

We would like to thank the anonymous referee for providing us with constructive comments and suggestions.

This work has been partially supported by Università di Roma La Sapienza (Dipartimento di Fisica, Gruppo SCAE).

\section{REFERENCES}

Becker, P. A., Le, T., \& Dermer, C. D. 2006, The Astrophysical Journal, 647, 539

Goodman, L. 1962, Journal of the American Statistical Association, 57, 54

Jones, F. C. 1968, Physical Review, 167, 1159

Kardashev, N. S. 1962, Soviet Astronomy, 6, 317

Katarzyński, K., Ghisellini, G., Mastichiadis, A., Tavecchio, F., \& Maraschi, L. 2006, Astronomy and Astrophysics, 453, 47

Lemoine, M., \& Pelletier, G. 2003, The Astronomical Journal, 589 L73

Massaro, E., Perri, M., Giommi, P., \& Nesci, R. 2004, Astronomy and Astrophysics, 413, 489

Massaro, E., Tramacere, A., Perri, M., Giommi, P., \& Tosti, G. 2006, Astronomy and Astrophysics, 448, 861

Massaro, F., \& Grindlay, J. E. 2011, arXiv, astro-ph.HE

Massaro, F., Tramacere, A., Cavaliere, A., Perri, M., \& Giommi, P. 2008, Astronomy and Astrophysics, 478, 395

Melrose, D. B. 1969, Astrophysics and Space Science, 5, 131

Moderski, R., Sikora, M., Coppi, P. S., \& Aharonian, F. 2005, Monthly Notices of the Royal Astronomical Society, 363, 954

O'Sullivan, S., Reville, B., \& Taylor, A. M. 2009, Monthly Notices of the Royal Astronomical Society, 400, 248
Park, B. T., \& Petrosian, V. 1996, Astrophysical Journal Supplement v.103, 103, 255

Peacock, J. A. 1981, Royal Astronomical Society, 196, 135

Ramaty, R. 1979, In: Particle acceleration mechanisms in astrophysics; Proceedings of the Workshop, 56, 135, a\&AA ID. AAA027.078.006

Rybicki, G. B., \& Lightman, A. P. 1986, Radiative Processes in Astrophysics, iSBN: 0-471-82759-2

Skilling, J. 1975, Royal Astronomical Society, 172, 557, a\&AA ID. AAA014.143.004

Stawarz, L., \& Petrosian, V. 2008, The Astrophysical Journal, 681, 1725

Tramacere, A. 2007, Ph.D. Thesis, Spectral Variability in Blazar's High Energy Emission, La Sapienza University, Rome, 1

Tramacere, A., Giommi, P., Perri, M., Verrecchia, F., \& Tosti, G. 2009, Astronomy and Astrophysics, 501, 879

Tramacere, A., Massaro, F., \& Cavaliere, A. 2007, Astronomy and Astrophysics, 466, 521 